\title{
Pre-stack full waveform inversion of ultra-high-frequency marine seismic reflection data
}

\author{
Giuseppe Provenzano ${ }^{\star}$, Mark E. Vardy ${ }^{\dagger}$, Timothy J. Henstock ${ }^{\star}$
}

\section{SUMMARY}

The full waveform inversion $(F W I)$ of seismic reflection data aims to reconstruct a detailed physical properties model of the subsurface, fitting both the amplitude and traveltime of the reflections generated at physical discontinuities in the propagation medium. Unlike reservoirscale seismic exploration, where seismic inversion is a widely adopted remote characterisation tool, ultra high frequency $(U H F, 0.2-4.0 \mathrm{kHz})$ multi-channel marine reflection seismology is still most often limited to a qualitative interpretation of the reflections' architecture. Here we propose an elastic full waveform inversion methodology, custom-tailored for pre-stack UHF marine data in vertically heterogeneous media to obtain a decimetric-scale distribution of Pimpedance, density and Poisson's ratio within the shallow sub-seabed sediments. We address the deterministic multi-parameter inversion in a sequential fashion. The complex trace instantaneous phase is first inverted for the P-wave velocity to make-up for the lack of low-frequency in the data and reduce the non-linearity of the problem. This is followed by a short-offset Pimpedance optimisation and a further step of full offset range Poisson's ratio inversion. Provided that the seismogram contains wide reflection angles ( $>40$ degrees), we show that it is possible to invert for density and decompose a-posteriori the relative contribution of P-wave velocity and density to the P-impedance. A broad range of synthetic tests is used to prove the potential of the methodology and highlights sensitivity issues specific to UHF seismic. An example application to real data is also presented. In the real case, trace normalisation is applied to minimise the systematic error deriving from an inaccurate source wavelet estimation. 
The inverted model for the top 15 meters of the sub-seabed agrees with the local lithological information and core-log data. Thus we can obtain a detailed remote characterisation of the shallow sediments using a multi-channel sub-bottom profiler within a reasonable computing cost and with minimal pre-processing. This has the potential to reduce the need of extensive geotechnical coring campaigns.

Key words: Sub-metric resolution seismic - Ultra High Frequency seismic - Elastic FWI Multiparameter inversion - Sediment characterisation.

\section{INTRODUCTION}

A quantitative physical model of near-surface marine sediments is of crucial importance in a broad range of environmental and engineering contexts, from the assessment of tsunamigenic landslides hazard and offshore structure stability, to the identification and monitoring of gas storage sites. Currently, marine sediment characterisation is heavily reliant on direct sampling of the seabed, using cores, borehole and/or cone penetrometers (CPTUs) (e.g. Stoker et al., 2009; Vanneste et al., 2012). In this framework, marine seismic reflection data is limited to providing information about the architecture of the stratigraphic discontinuities generating the reflections in the sub-surface, combined with a predominantly qualitative interpretation of the relative amplitude and polarity of the seismic phases. The structural information derived from the sub-bottom profiling can be correlated to core or borehole logs, where possible, to extend the geotechnical/lithological data from the sampling sites across larger basins. In laterally heterogeneous areas, a large number of direct samples are required to reconstruct the spatial variation of the model to the degree of accuracy required by engineering applications and such an approach is expensive and time-consuming. The reliability of a quantitative estimation of sediment properties is also likely to be undermined by the coring process itself, which deforms and mechanically alters the sample, particularly in low-effective stress environments.

Even though computationally demanding, the inversion of ultra high frequency $(0.2-4.0 \mathrm{kHz}$,

\footnotetext{
* Ocean and Earth Science, National Oceanography Centre Southampton, University of Southampton, European Way, Southampton, SO14 3ZH

$\dagger$ Marine Geosciences Group, National Oceanography Centre, Southampton, European Way, Southampton, SO14 3ZH
} 
$U H F$ ) seismic reflection data potentially provides a non-destructive, faster and cheaper alternative to characterise the mechanics of the sub-seabed. The quantitative interpretation of pre-stack seismic data is a well-established and widely accepted procedure in industry and basin-scale exploration, in the form of either full waveform inversion (FWI) (Tarantola, 1984) or reflection amplitude versus offset inversion (AVO) (Ruthenford \& Williams, 1989). It allows improved imaging of complex structures (Tarantola, 1984; Mora, 1980; Virieux \& Operto, 2009), detailed rock physics characterisation of oil and gas reservoir (Ostrander, 1984; Ruthenford \& Williams, 1989; Fatti et al., 1994; Mallick \& Adhikari, 2015), and enhanced resolution regional geology models (Gulick et al., 2013; Morgan et al., 2013).

Over the last few years, quantitative interpretation techniques have started to be applied also to near-surface seismic data in order to remotely derive decimetric resolution shallow sediment physical properties in terms of reflection coefficient and acoustic quality factor (Bull et al., 1998; Pinson et al., 2008; Vardy et al., 2012; Cevatoglu et al., 2015). Holland \& Dettmer (2013) used the angle-dependent reflection amplitude as a function of frequency to derive physical properties layering and gradients within the shallow sediments. Recently, post-stack acoustic inversion has been successfully applied on ultra-high-frequency seismic data (Vardy, 2015) to derive quantitative sediment properties from the acoustic impedance.

The dependancy of a pre-stack seismic gather on the elastic properties of the propagation medium theoretically allows us to obtain a detailed distribution of compressibility, shear properties and density to the scale of a fraction of the propagated wavelength. Although such properties can be retrieved through the inversion of the reflections' AVO, a full waveform approach has the advantage to account for all the wave phenomena (Tarantola, 1984, 1986; Fichtner, 2011), within the required resolution and modelling approximation. By exploiting the information contained in the complete waveform, FWI outperforms AVO inversion in most realistic reservoir geophysics application (Mallick \& Adhikari, 2015), especially when complicated layer interference and velocity gradients are present (Xu et al., 1993; Igel et al., 1996), which is likely to be a factor in UHF near-surface seismic data.

Here we invert the full waveform of UHF marine data in order to obtain a sub-metric resolution 
G. Provenzano et al.

elastic model of the near-seabed. Tests on both synthetic and real pre-stack data, demonstrate the capability of the method to obtain a detailed characterisation of the medium in terms of independent estimates of P-wave velocity, density and Poisson's ratio.

\section{METHODOLOGY AND SYNTHETIC EXAMPLES}

\subsection{Full marine seismogram modelling in the varying streamer depth case}

The inversion of pre-stack marine seismic data is often addressed within the acoustic approximation to obtain a detailed pressure wave velocity model (Fichtner, 2011; Virieux \& Operto, 2009; Tarantola, 1984). Although this approach is widely employed in both industry and academia as an effective tool to improve the quality of the seismic imaging (Morgan et al., 2013), acoustic waveform inversion fails to reproduce an accurate model of the subsurface when shear properties vary in the subsurface, or density is not correlated to P-impedance variations, creating significant amplitude and phase versus offset effects (Mallick \& Adhikari, 2015; Silverton et al., 2015). The acoustic approximation is usually justified by the unaffordable computational cost of the finitedifferences or finite elements modelling in laterally varying elastic media.

In this paper we account for the elastic properties, assuming that the medium's heterogeneity can be realistically approximated as purely vertical in the range of the imaging aperture (Virieux \& Operto, 2009); in UHF seismic, this would be in the order of tens of meters. Despite an inherent loss of horizontal resolution, such an assumption is acceptable in shallow, recent and weakly tectonised sediments, and allows for the forward model to be computed using an analytic fast solution in the plane wave domain within a reasonable computational cost (Fuchs \& Müller, 1971). The program chosen to compute the pressure seismograms is the Ocean Acoustics and Seismic Exploration Synthesis from MIT (Schmidt \& Jensen, 1985; Schmidt \& Tango, 1986.), which addresses the reflectivity modelling in an efficient and accurate way for the frequency-wavenumber range of interest.

In UHF marine reflection seismic data, receiver depths in the order of a few meters produce receiver ghost reflections that correspond to frequency notches inside the bandwidth of the signal. Significantly sagging streamer geometries are often observed in the marine-lacustrine setting typ- 
ical of UHF seismic (Pinson, 2009) and this sub-metric to metric scale variations of the streamer geometry as a function of offset cause non-negligible changes in the source-acquisition system impulse-response. Furthermore, the sea-surface topography approaches the seismic wavelength of a UHF wavefield, causing the sea-surface reflection coefficient to change significantly across the streamer length. In this work we chose to include these factors in the computation of the synthetic seismograms, as opposed to a deconvolution on the observed data. Although inverse ghost filtering would yield a spectral whitening that can be beneficial to the seismic resolution, it is liable to create artefacts inside the bandwidth of the signal which could severely undermine the inversion performance. On the other hand, an explicit full wavefield modelling using the one-dimensional solver would require one forward computation per each receiver offset-depth couple in the appropriate wavenumber bandwidth; since the wavenumber ranges necessary to model each offset are largely overlapping, this approach is clearly inefficient and results in a non-affordable computing cost. In this work, we developed an efficient total seismogram modelling method, which requires only the computation of the pure up-going wavefield at one arbitrary receiver depth, and derives the whole gather in the frequency-wavenumber domain using wavefield decomposition (Verschur et al., 1992; Aytun, 1999). For each channel, the prediction of the down-going wavefield and the downward propagation in the plane wave domain are implemented as a linear filter with the estimated receiver depth and sea-surface reflection coefficient; an inverse two-dimensional Fourier transform gives a seismic gather in an expanded offset range from which the trace at the appropriate offset is selected. The final predicted seismogram is then obtained by merging the different offsets. The alternative proposed here allowed for a reduction of the computing time of one order of magnitude. Details on the theory and the implementation of the method are given in Appendix A.

\subsection{Gauss-Newton seismic inversion}

FWI is a non-linear and ill-posed parameter estimation technique, which iteratively updates the earth model $\mathbf{m}$ by minimising a weighted measure of the difference between the computed and recorded seismic data $\delta \mathbf{d}(\mathbf{m})$ (Tarantola, 1984; Virieux \& Operto, 2009; Fichtner, 2011). The objective or misfit functional accounts for the amplitude and phase characteristics of the wavefield, 
either as in the full seismogram, or extracted as pre-stack attributes (Fichtner, 2011; JimenezTejero et al., 2015) and it is regularised in order to penalise physically non-meaningful solutions (Menke, 1989; Asnaashari et al., 2012). The least square regularised objective function reads:

$e(\mathbf{m})=\delta \mathbf{d}^{T} \mathbf{W}_{\mathbf{d}}^{T} \mathbf{W}_{\mathbf{d}} \delta \mathbf{d}+\delta \mathbf{m}^{T} \mathbf{W}_{\mathbf{m}}^{T} \mathbf{W}_{\mathbf{m}} \delta \mathbf{m}$

where $\mathbf{W}_{\mathbf{d}}$ and $\mathbf{W}_{\mathbf{m}}$ are respectively the data and model covariance matrix and $\delta \mathbf{m}$ is measured with respect to a reference model.

At each iteration, the current model $\mathbf{m}_{i}$ is updated in the direction of the negative gradient of the misfit functional, scaled and weighted by the inverse Hessian matrix $\mathbf{H}$ :

$\mathbf{m}_{i+1}=\mathbf{m}_{i}-\mathbf{H}\left(\mathbf{m}_{i}\right)^{-1} \nabla e\left(\mathbf{m}_{\mathbf{i}}\right)$

The ascent direction $\nabla e$ is the scalar product between the wavefield partial derivative matrix ( $\mathrm{Ja}$ cobian or sensitivity matrix $\mathbf{J}$ ), and the data residual vector $\delta \mathbf{d} ; \mathbf{H}$ contains the zero-lag autocorrelation of the sensitivity matrix, plus a second order term depending upon the partial second derivatives of the wavefield with respect to each model parameter (Virieux \& Operto, 2009).

In three-dimensional FWI, the number of independent model parameters makes the computation of the partial derivative wavefield most often unaffordable. To overcome this limitation, the model update direction is efficiently computed using the adjoint state method (Lailly, 1983; Tarantola, 1984; Virieux \& Operto, 2009), whereas the inverse Hessian in equation 2 can be replaced by a line-search estimate of the optimal step-length, which ensures the convergence towards the nearest local minimum (Nocedal \& Wright, 2006; Virieux \& Operto, 2009). However, such a steepest descent implementation does not account for the scaling and uncoupling effect of the inverse Hessian (Virieux \& Operto, 2009; Operto et al., 2013) and a robust estimate of the latter in fact significantly improves parameter resolution and convergence speed (Pratt et al., 1998; Operto et al., 2013). Quasi-newton methods, such as the lBFGS (Malinkowski et al., 2011; Gholami et al., 2013; Dagnino et al., 2014), are now a commonplace implementation of Hessian-based FWI, in which the inverse Hessian is recursively estimated from the evolution of the gradient and model update over a number of previous iterations (Nocedal \& Wright, 2006; Virieux \& Operto, 2009). 
Sheen et al. (2006) and Shin et al. (2001), on the other hand, propose to reduce the computational burden of the partial derivative wavefield by exploiting the source-receiver reciprocity.

In this paper, the waveform inversion is implemented as a damped least square Gauss-Newton optimisation problem (Menke, 1989; Aster et al., 2005). In the Gauss-Newton method, a locally linear misfit functional is assumed (Kormendi \& Dietrich, 1991; Menke, 1989; Aster et al., 2005), which allows the second order term of the Hessian to be dropped (Virieux \& Operto, 2009). We obtain explicitly the sensitivity matrix $\mathbf{J}$ by perturbing each model parameter at each layer depth; the resulting partial derivative wavefield is propagated from the secondary virtual sources to the receivers' position (Rodi, 1976; Sheen et al., 2006; Operto et al., 2013). The effectiveness of this approach in scaling and weighting the gradient is higher than the steepest-descent and quasi-Newton methods, because the approximate Hessian $\mathbf{J}^{T} \mathbf{J}$ is computed rather than statistically estimated. The relatively low number of unknowns of the 1D modelling makes the computing cost affordable, with wide scope for improvement thanks to the highly parallelisable nature of the sensitivity matrix. The Gauss-Newton method can be applied to trace-normalised seismic data (Lee \& Kim, 2003), in which the non-physical phase correction resulting as a by-product of the source deconvolution makes the back-propagation of the adjoint-field inappropriate (Virieux \& Operto, 2009). Also, the presence of strong receiver ghost reflections in UHF data undermines the accuracy of the reverse time migration of the residuals (Sun et al., 2015). The model update $\delta \mathbf{m}$ at each iteration can therefore be expressed as:

$\delta \mathbf{m}=\left(\mathbf{J}^{T} \mathbf{W}_{\mathbf{d}} \mathbf{J}+\mathbf{W}_{\mathbf{m}}\right)^{-1} \mathbf{J}^{T} \mathbf{W}_{\mathbf{d}} \delta \mathbf{d}$

The first factor of the right hand side of Eq. 3 is the regularised approximate Hessian, while the second is the gradient of the misfit functional. Eq. 3 has the form of the regularised least square inverse solution for a problem of the kind:

$\delta \mathbf{d}=\mathbf{J} \delta \mathbf{m}$

It is therefore possible to express the linear operator $\mathbf{J}$ mapping the data residual $\delta \mathbf{d}$ from the data space into the model update $\delta \mathbf{m}$ space in the Singular Value Decomposed (SVD) domain. If, for 
the sake of simplicity, we set the data covariance matrixes equal to the identity and scale the model covariance by the factor $\alpha$, Eq. 3 takes the form (Aster et al., 2005):

$\delta \mathbf{m}=\sum_{i=1}^{k} \frac{s_{i}{ }^{2}}{s_{i}{ }^{2}+\alpha^{2}} \frac{\mathbf{U}_{(:, i)}{ }^{T} \delta \mathbf{d}}{s_{i}} \mathbf{p}^{T} \mathbf{V}_{(:, i)}$

where $\mathbf{U}$ is the data eigenvector matrix, $s_{i}$ is the $i_{t h}$ singular value and $\mathbf{V}$ is the model eigenvector matrix. This equation expresses the model update vector as the result of the projection of the data residual vector on the model update vectorial space. The hyper parameter $\alpha$ contributes in the filter factor $\frac{s_{i}{ }^{2}}{s_{i}{ }^{2}+\alpha^{2}}$ to damp out the small singular value responsible for numerical instability (Menke, 1989; Aster et al., 2005; Asnaashari et al., 2012). The preconditioning vector $\mathbf{p}$ assigns a different relative weight to each parameter of the sensitivity matrix in order to guide the inversion towards geologically plausible solutions.

From a physical point of view, Eq. 4 is equivalent to the Born approximation of the wavefield (Jannane, 1989; Virieux \& Operto, 2009; Fichtner, 2011), which implies that the data residuals are linearly related to missing heterogeneities in a background elastic model (Tarantola, 1984; Jannane, 1989; Virieux \& Operto, 2009; Fichtner, 2011). In this framework, in order for the convergence to local minima to be prevented, the background velocity distribution needs to account for the traveltime information of the data within half a propagated wavelength, otherwise cycle skipping (Virieux \& Operto, 2009) occurs and a spurious solution is obtained. Resuming, the inversion process involves the following steps:

(i) Computation of the seismogram for the current model $\mathbf{m}_{i}$;

(ii) Computation of the residual vector $\delta \mathbf{d}$;

(iii) Computation of the sensitivity matrix by perturbing each model parameter and computing the residuals in a forward finite difference scheme;

(iv) Singular value decomposition and generalised inverse computation;

(v) Computation of the model update $\delta \mathbf{m}$ and of the model $\mathbf{m}_{(i+1)}$;

(vi) Seismogram computation for the $\mathbf{m}_{(i+1)}$ model;

(vii) Misfit computation;

(viii) If the convergence criteria are satisfied, the inversion ends, otherwise goes back to (ii). 
The process stops when a maximum number of iteration is reached, the misfit goes below a threshold, or the misfit evolution function has reached a plateau.

\subsection{A strategy for the multi-parameter problem}

An isotropic and elastic medium is univocally described by a spatial distribution of three independent parameters (Aki \& Richards, 2002), most commonly density and the Lamè coefficients (Tarantola, 1986); although equivalent in a forward modelling sense, different parametrisations have different convergence properties and parameters' resolution. The most desirable parametrisation guarantees the minimum crosstalk among the unknowns of the inversion (Tarantola, 1986; Kormendi \& Dietrich, 1991); ideally, the partial derivative wavefield of one parameter should be uncorrelated with the residual wavefield produced by each other independent parameter (Tarantola, 1986; Operto et al., 2013). In marine reflection seismic data, the presence of only one propagation mode impedes the opportunity to obtain independent estimates of $\mathrm{P}$-wave $(V p)$ and $\mathrm{S}$-wave velocity (Vs) (Jin et al., 1992; Igel et al., 1996); on the other hand, density is strongly coupled with P-wave velocity at narrow reflection angle; the two parameters can't be effectively resolved and in fact yield a posterior reconstruction of the P-impedance model (Tarantola, 1986; Operto et al., 2013). Here we choose to parametrise the reflectivity of the earth model as a distribution of P-impedance, Poisson's ratio and density (Debski \& Tarantola, 1995; Igel et al., 1996), super-imposed to a long-wavelength P-wave velocity model that controls the wavefield kinematic (Tarantola, 1986; Jannane, 1989).

The limited offset, limited bandwidth, lack of diving waves and multi-component data of UHF data produce a highly hierarchical dependancy on the multi-parameter space as a function of the reflection angle range (Tarantola, 1986). P-impedance is the dominant parameter over the whole angle range, and it is sufficient to explain the reflected energy at near-zero reflection angle (Tarantola, 1986). A second order contribution to the wavefield energy is given by the variation of the reflection amplitude with angle; this depends upon the Poisson's ratio and density contrast at the layer interfaces, the first dominating the mid offset AVO, the latter having an increasing importance at greater reflection angles (Koefoed, 1955; Ostrander, 1984; Ruthenford \& Williams, 1989; 
Mallick \& Dutta, 2002). The resulting Hessian matrix for a scatterer layer is highly rank-deficient, meaning that only one parameter class can be effectively inverted for in a simultaneous multiparameter inversion (Operto et al., 2013); despite the inherent stability of the SVD approach, this makes the multiplication by the inverse Hessian not ideal as a means to uncouple the different components of the gradient. On the other hand, the angle dependancy of the secondary virtual sources for the chosen parametrisation, allows us to orthogonalise the problem by appropriately weighting the residual wavefield and inverting sequentially each independent parameter, from the strongest to the weakest contributor to the residual energy (Tarantola, 1986; Igel et al., 1996; Operto et al., 2013). This approach has also the advantage of a simpler implementation of the regularised least-square inversion, as it does not require to build a model covariance matrix that balances the contribution of parameters with different weights (Eq. 3).

We first invert for P-wave velocity by keeping Poisson's ratio and density constant to the background values, windowing the data and the partial derivative field around the near-offsets; this is equivalent to a P-impedance inversion, for density is kept constant. We then invert the whole offset range for Poisson's ratio; the virtual source radiation patterns of Poisson's ratio and density are partially overlapping in the mid reflection angle range, hence the estimate will be to some extent affected by crosstalk. If wide reflection angles ( $>40$ degrees) are available in the data, the relative contributions of density and P-wave velocity to P-impedance are decomposed by inverting for density at constant P-impedance. A cyclical update of density and Poisson's ratio in this stage makes up for the effect of crosstalk resulting from the previous stage. The reliability of the a-posteriori $\mathrm{P}$-wave velocity estimate is ensured by the broad wavenumber content of the P-impedance model (Operto et al., 2013), as opposed to a a-posteriori estimation of density from a [P-impedance, Vp, Poisson's ratio] parametrisation.

\subsection{Test on synthetic UHF marine reflection data}

We test the performance of the sequential inversions strategy on a synthetic example of pre-stack UHF data. In this experiment, the starting P-wave velocity model is accurate enough to allow for an effective inversion of the reflectivity of the medium. We also assume that the source wavelet is 
perfectly known. The synthetic true model has 18 layers, for a total thickness of 12 meters below the sea-bottom interface at 15 meters water depth; it includes $20 \mathrm{~cm}$ thick target horizons with distinct elastic characteristics. Changes in acoustic impedance are either correlated to variation in both density and velocity, or density only, and the Poisson's ratio varies independently with respect to the P-wave velocity, in order to mimic both changes in fluid saturation in the same lithology and lithological stratifications (Ruthenford \& Williams, 1989). The perfect elasticity implies that there is no intrinsic attenuation (Aki \& Richards, 2002). The theoretical source signature is a minimum phase Boomer wavelet (Fig. 1) band-pass filtered 0.1-1.5 kHz (almost 4 octaves). The acquisition simulates a source depth in the order of 10 centimetres and a 60 channel streamer with a group spacing of 1 meter and a minimum offset equal to 13 meters. The receiver depth is equal to 1.85 meters, which corresponds to receiver ghost notches at multiples of $400 \mathrm{~Hz}$ (Aytun, 1999). The true data have been contaminated by a band-limited zero-mean gaussian noise, for a signal-tonoise ratio equal to 50 with respect to the amplitude of the strongest reflection.

The starting model of the iterative inversion is a low-pass filtered ( 2 meters cut-off wavelength) version of the true model, containing the correct velocity and density trend, but accounting only for the sea-floor primary reflection traveltime and surface-related multiples. With the exception of the sea-floor, the location of the discontinuities is unknown. The starting model has been parametrised as a stack of 58 homogeneous, elastic and isotropic layers with a thickness chosen to be in the order of the tuning thickness for the frequency band employed and the expected velocity values $(\lambda / 4)$. This results in a model space with 174 degrees of freedom, which are broken down to 58 per stage in the sequential inversion.

In Stage 1 ( Fig. 2) the data are inverted for P-wave velocity only. An accurate P-impedance profile is retrieved and a good waveform match is attained in the short offsets of the seismic gather. Note how the $20 \mathrm{~cm}$ thick low-density bed at 3.4 meters is incorrectly identified by a drop in P-wave velocity, while in layers where P-wave velocity and density are correlated, the P-wave velocity change is overestimated to explain the P-impedance variation. This confirms the considerations about the [Vp, density, Poisson's ratio] parametrisation in the reflection regime 
(Tarantola, 1986; Operto et al., 2013). The Poisson's ratio is kept constant in this stage, so that not only P-wave, but also S-wave velocity is effectively updated.

At Stage 2 the data are inverted for Poisson's ratio, while the other parameters are kept fixed. The misfit is here dominated by the reflection amplitude versus offset variation. The inversion updates the model in the correct direction, but crosstalk with density prevents from reconstructing the fine-scale details of the shear properties, especially where the relative contribution of P-wave velocity and density to the P-impedance is poorly described ( Fig. 3).

Stage 3 of the inversion firstly optimises density until an accurate impedance distribution is obtained. At this point, the final parametrisation [P-impedance, density, Poisson's ratio] is effectively adopted and density evolves independently from P-impedance; this produces a consequent change in P-wave velocity. Note how the P-impedance contrast at 3.4 meters is now correctly decomposed in its relative P-wave velocity and density contributions. The effect crosstalk between Poisson's ratio and density in the previous stage is also made up for by cyclically updating the two parameters: the effectiveness of this stage is particularly evident in layers where the underestimate of density in the starting model had induced an underestimate of Poisson's ratio (and vice-versa). The final model (Fig. 4) is a high-fidelity representation of the elastic model.

The robustness of the proposed inversion strategy can be tested against variations of the signalto-noise ratio $(S N R)$. Gaussian band-limited noise with zero mean has been added in the signal frequency band to the field data with different SNRs and the normalised $L 2$ model misfit has been computed per each parameter class. As shown in Fig. 5, P-impedance is the most robust parameter to increasing random noise energy in the data; Poisson's ratio is also remarkably robust in a broad range of SNR, while the results of the P-wave velocity/density decomposition at constant impedance quickly deteriorates with decreasing signal-to-noise ratio. Note how, for signalto-noise ratio lower than 50, the Poisson's ratio data misfit increases steeply, arguably because of the crosstalk between density and shear properties. The results are consistent with the idea that shear properties and density are second order contributions to the misfit value in marine reflection seismograms (Tarantola, 1984, 1986), hence their effect is more quickly buried in high noise levels. Nevertheless, as we will see in the next section, general conclusions about the robustness 
of the parameter estimation need to account for the medium characteristics and the acquisition parameters.

\section{SENSITIVITY ISSUES SPECIFIC TO UHF DATA}

The elastic full waveform inversion of UHF reflection data suffers from specific factors, which are discussed in detail in this section:

(i) The limited reflection angle due to the short streamers normally employed limits the possibility to obtain independent information about P-wave velocity and density.

(ii) The inversion for the Poisson's ratio distribution is highly non-unique, because the AVO characteristics of the gather are dependent upon the Poisson's ratio contrasts (Aki \& Richards, 2002), rather than its absolute value, so that the misfit function is insensitive to shear properties gradients. Furthermore, the high $\mathrm{Vp} / \mathrm{Vs}$ ratio of the shallow marine sediments (in the order of $10^{1}-10^{2}$ ) spans a very narrow Poisson's ratio range (Hamilton, 1970).

(iii) The sensitivity in the mid to long wavelengths of the P-wave velocity model is poor because of the lack of low-frequencies and the limited available offsets (Mora, 1980; Jannane, 1989).

(iv) The accuracy of the UHF source wavelet estimation using traditional methodologies is compromised by the characteristics of the data and of the typical reflectivity series involved.

\subsection{Sensitivity to constant-impedance density variations}

A broad literature about the parameter dependancy of marine seismic reflection data shows that independent high frequency variations of P-wave velocity and density at constant impedance are poorly constrained parameters when limited offset are available (e.g. Debski \& Tarantola, 1995; Jannane, 1989; Igel et al., 1996). We conduct a sensitivity analysis on a simple homogeneous model with a low impedance $50 \mathrm{~cm}$ thick layer, whose density is changed at constant impedance, as a function of the maximum reflection angle at the target. The relative $L 2$ data misfit is computed against a reference model and the results are plotted in decibel scale in Fig. 6a. The rapid decrease in the relative data misfit with decreasing reflection angle suggests that the sensitivity of the inversion to such changes in the subsurface is highly sensitive to the ratio between streamer aperture 
and target depth. In order to decompose the amplitude and traveltime contribution to the measured misfit, we compute the offset-dependent cross-correlation value and lag; the results show how a density perturbation at constant impedance produces a change in the maximum value of the cross-correlation function (Fig. 6b), whilst the lag is not significantly affected, being always equal to or lower than one sample. This results confirm that, although the reflection travel times are insensitive to such perturbations, being mostly controlled by a long-wavelength distribution of the P-wave velocity, density variations at constant impedance do affect the angle-dependent reflectivity of the propagation medium, as a second order contribution to the AVO reflection amplitude (Aki \& Richards, 2002). Three density profiles have been picked from the population of models used for the sensitivity analysis to test how this translates into the inversion results. We use a constant starting density model, where the impedance change at the target layer is entirely explained by a P-wave velocity variation, a situation comparable to the end of the second stage of the inversion. The Poisson's ratio profile of the starting model is correct, thereby crosstalk effects do not play a role in the test. The curves computed from the exact AVO equation (Aki \& Richards, 2002) for the starting and true models show how the inaccuracy in the density profile at constant impedance contrast produces a residual AVO at long offset, which is greater for the shallower model. The quality of the P-wave velocity/density decomposition in the final models is highly dependent on the reflection angle range (Fig. 7) and no independent information about P-wave velocity and density can be reliably obtained at reflection angles below 30 degrees. Model misfit for each parameter class in the 18 layers model (Fig. 8) as a function of a maximum reflection angle shows that P-impedance and Poisson's ratio are robust to changes in the reflection angle; in contrast, the P-wave velocity profile quickly deteriorates as the reflection angle range narrows.

\subsection{Sensitivity to Poisson's ratio in high $\mathrm{Vp} / \mathrm{Vs}$ media}

Changes in lithology, pore pressure and saturating fluids produce differential variations of compressibility and shear properties, determining changes on the ratio between $\mathrm{P}$-wave and $\mathrm{S}$-wave velocities (Vp/Vs) (Ruthenford \& Williams, 1989; Mallick \& Dutta, 2002; Igel et al., 1996). How- 
ever, the $V p / V s$ ratio controls the reflectivity of a medium via the Poisson's ratio (Koefoed, 1955; Mavko et al., 2009):

$\nu=\frac{\left(V_{p} / V s\right)^{2}-2}{2\left[\left(V_{p} / V s\right)^{2}-1\right]}$

The non linear relationship of Eq. 6 determines a range-dependent sensitivity to $V p / V s$ changes. For low $\nu$, small changes in the $V p / V s$ ratio head to significant variation of $\nu$, thereby creating important AVO effects; in contrast in the range from 0.45 to 0.5 , where normally most shallow marine sediments fall (Hamilton, 1970), large changes in $V p / V s$ correspond to small variation of Poisson's ratio (Fig. 9a), limiting the AVO response (Mallick \& Dutta, 2002).

A sensitivity analysis is performed on a simple elastic model with a low-impedance $50 \mathrm{~cm}$ thick target layer, whose $\mathrm{Vp} / \mathrm{Vs}$ ratio is 5 times higher than the surrounding homogeneous medium, simulating the presence of a under-consolidated, high-porosity and high pore-pressure sediment bed. Within a broad range of $\nu$, we introduce perturbations to the $\mathrm{S}$-wave velocity of the target layer and measure the relative $L 2$ misfit with respect to a reference model. The sensitivity to $V s$ perturbations rapidly falls with increasing Poisson's ratio, regardless the amount of perturbation added (Fig. 9b). To study how this loss of sensitivity affects the inversion performances, we pick four models from the sensitivity analysis in four different Poisson's ratio ranges and run the inversion starting from a homogeneous profile. Although the $V p / V s$ ratio contrast at the target layer boundary is 5 for every model, the corresponding Poisson's ratio variation drops quickly below $5 \%$ for $\mathrm{Vp} / \mathrm{Vs}$ ratio higher than 4 (Table 1). This is apparent in the residual Amplitude Versus Angle (AVA) computed between the starting homogeneous Poisson's ratio model and the true model containing the target interface (Fig. 10). As a consequence, in the presence of noise the match between retrieved and true S-wave velocity profile deteriorates when the Poisson's ratio background value increases (Fig. 11). The $V p / V s$ increase at the target interface is accommodated for by a $V s$ profile with a similar contrast, but an erroneous relatively long-wavelength trend.

To address this non-uniqueness issue, we propose a structure-constrained gradient preconditioning: in the framework of a sequential strategy, the normalised derivative of the P-impedance obtained from the first stage of the inversion is used to weigh each element of the misfit gradient in the Poisson's ratio stage. Thus the inversion updates preferentially the Poisson's ratio of layers 


\begin{tabular}{ccccc}
\hline & $\nu 1$ & $\nu 2$ & $\mathrm{Vp} / \mathrm{Vs} 1$ & $\mathrm{Vp} / \mathrm{Vs} 2$ \\
\hline $\mathrm{a}$ & 0.33 & 0.4949 & 2 & 10 \\
\hline $\mathrm{b}$ & 0.4666 & 0.4987 & 4 & 20 \\
\hline $\mathrm{c}$ & 0.4920 & 0.4996 & 8 & 40 \\
\hline $\mathrm{d}$ & 0.4984 & 0.4999 & 18 & 90
\end{tabular}

Table 1. Table of Poisson's ratio and Vp/Vs ratio of the models used for the sensitivity analysis. Index 1 refers to the surrounding medium, index 2 to the target layer.

with a significant change in the acoustic properties, imposing a correlation between changes in P-impedance and $V p / V s$ ratio. The final model misfit is significantly improved by preconditioning (Fig. 12), especially for the low-sensitivity, high Poisson's ratio cases. Ambiguities in the interpretation of the interface location are reduced without the need of additional a-priori information. This approach remains essentially data-driven and fits naturally in the sequential inversion strategy.

\subsection{Complex trace inversion to improve the model kinematics}

The lack of low frequencies and the limited offsets in UHF data determine a low sensitivity to the mid-to-low wavelengths of the earth-model (Mora, 1980; Jannane, 1989). Although the background P-wave velocity distribution within the top 50 meters of the sub-seabed is close to the water velocity (Hamilton, 1970), this characteristic can be conducive to cycle-skipping and local minimum entrapment in the P-wave velocity inversion (Tarantola, 1984; Virieux \& Operto, 2009), which would jeopardise the reconstruction of the elastic model in the following steps. To reduce this problem, when the knowledge of the starting model is poor, we exploit the properties of the complex trace, combined with a multi-scale approach, which progressively includes higher frequency in the inversion (Bunks et al., 1995). The seismic signal $s(t)$ can be considered as the real part of a complex $c(t)$ trace whose imaginary part is its Hilbert transform $h(t)$ (Taner et al., 1979), i.e. its $\pi / 2$ phase-shifted version:

$\mathbf{c}(t)=\mathbf{d}(t)+i \mathbf{h}(t)$ 
The instantaneous phase (IP) is defined as the inverse tangent of the ratio between the imaginary and the real part, while the modulus of the complex trace is known as the envelope or reflection strength. Here we use a modified version of the instantaneous phase, proposed by Jimenez-Tejero et al. (2015), where the inverse tangent computed on the ratio between the absolute value of the imaginary trace and the real trace. It reads:

$\phi(t)=\tan ^{-1}\left(\frac{|\mathbf{h}(t)|}{\mathbf{d}(t)}\right)$

Separating instantaneous amplitude and phase information of the seismogram is beneficial to the convergence properties of FWI (Fichtner, 2011; Bozdag et al., 2011; Fichtner et al., 2008). The modified instantaneous phase is less liable to cycle skipping when FWI is applied to limitedoffset, band-limited seismic reflection data (Jimenez-Tejero et al., 2015), which is commonly the case in near-surface marine reflection seismic; furthermore, the phase-only virtual source for Pwave velocity is naturally uncoupled in the reflection regime with Poisson's ratio and density over the whole offset range, for it is less AVO-dependent. For elastic seismic inversion, the complex trace attributes are easily incorporated into a multi-stage approach, where the instantaneous phase is inverted first for the P-wave velocity distribution until convergence is reached and the reflected phases are correctly aligned; after that, the elastic model parameters are inverted for using a misfit functional based on the instantaneous amplitude. The same considerations about the sensitivity to the elastic model space hold in the envelope-based misfit functional, as the trace envelope is sufficient to describe the AVO characteristics of the gather.

\subsection{The source-independent approach}

Inaccuracies in the estimation of the source wavelet are among the main causes of systematic error in the FWI of real data. Statistical source estimation based on the seismic convolution theory assumes a white reflectivity series (Dey \& Lines, 1998; Mallick \& Adhikari, 2015); although this is the most popular approach in seismic modelling and imaging, such an assumption is likely to be violated in UHF near-surface data, because the short reflectivity series involved do not allow for a statistically robust estimate of a white signal and graded boundaries become increasingly important at the sub-metric scale. More complex methods, such as homomorphic deconvolution in 
the cepstral domain (Ulrych, 1971), are compromised by strong ghost contamination. Therefore we use the source normalisation scheme proposed by Lee \& Kim (2003). According to the convolution theory, a seismic trace can be expressed in the frequency domain by multiplying the complex spectra of the source and the impulse response of the propagation medium, or Green's function (Aki \& Richards, 2002).

$\mathbf{d}(\omega)=\mathbf{s}(\omega) \mathbf{g}(\omega)$

Let $\mathbf{d}_{R}(\omega)$ be a reference trace chosen from the multi-channel seismic gather, where $\mathbf{g}(\omega)$ in Eq. 9 is replaced by $\mathbf{g}_{R}(\omega)$. Normalising the seismogram for the reference trace in the frequency domain effectively deconvolves the data from the source signature:

$\mathbf{d}_{\mathbf{N}}(\omega)=\frac{\mathbf{s}(\omega) \mathbf{g}(\omega)}{\mathbf{s}(\omega) \mathbf{g}_{R}(\omega)}=\frac{\mathbf{g}(\omega)}{\mathbf{g}_{R}(\omega)}$

where $\mathbf{g}_{R}(\omega)$ is the impulse response at the reference receiver. In Eq. 10, $\mathbf{d}_{N}$ is independent from the source signature and essentially reduced to the Green's function normalised to the impulse response at the reference receiver location, normally chosen to be the closest to the source (Lee \& Kim, 2003; Joo et al., 2012; Kwon et al., 2015). In the so-obtained normalised data space, the inversion, rather than being driven by the recorded wavefield, seeks for the match between the relative changes in the impulse response of the medium with respect to the reference trace position.

No changes in the virtual sources radiation patterns are observed, hence the parameter dependancy of the normalised data from the elastic model space is not modified. The spectral normalisation, however, is liable to instabilities if the reference trace spectrum contains near-zero values at certain frequencies. In order to overcome this issue, the back transformation to the time domain is computed assigning a specific weight to each frequency component to damp out the spikes in the spectra corresponding to poles in the inverse filter. Although this approach would be easily incorporated in a frequency domain inversion, back transformation to the time domain is necessary to allow for the inversion to be performed on the time-dependant complex trace attributes. 


\section{APPLICATION TO A REAL UHF DATASET}

\subsection{Setting and Data overview}

In order to test the performance of the proposed inversion scheme, we apply the methodology to a real UHF multi-channel shallow water seismic gather acquired in the Solent, UK. The seismic source is an electro-acoustic Boomer plate, capable of producing a high-frequency, wide-band and highly repeatable minimum phase pulse (Verbeek \& McGee, 1995) (Fig. 1); the receiver streamer has 60 channels with minimum offset equal to 10 meters, one-meter group spacing and 7 elements per group. Both source and receiver directivity filters have been applied to the synthetics in the $F K$ domain prior to the misfit computation (Verbeek \& McGee, 1995; Riedel \& Theilen, 2001). The inversion is performed on the reference-trace deconvolved seismograms, which is expected to be robust against inaccuracies in the wavelet estimate. Since the sea surface multiple reflections are not included in the forward modelling, the data are bottom muted above the first order sea floor multiple; the only other pre-processing applied to the data is the muting of the direct wave and a frequency filtering in the appropriate modelling band.

Previous geophysical data available in the area comprise acoustic quality factor $Q p$ (Pinson et al., 2008), and long-wavelength P-wave velocity field from Migration Velocity Analysis (MVA) (Pinson, 2009), along with lithology, porosity and density log-measurements of a proximal sediment core. Seismic to log calibration identifies a 1-to-3 meters thick gravel layer, with low $Q p$ (c. 50), high P-wave velocity and density; it overlays, separated by an erosive unconformity, an over-consolidated clay-dominated layered sediment sequence, with high $Q p$ (c. 150) and acoustic velocity. The sediment sequence below the unconformity is interbedded by sub-metric shelly and limestone layers, and thus likely has detectable variations of shear properties. At the bottom of this 15 meters thick sequence, there is a strong impedance contrast interface.

The combination of shallow water environment (16 meters water depth) and streamer aperture, makes the reflection angle range wide enough to obtain independent estimates of density and Pwave velocity (64 to 47 degrees from the shallowest to the deepest interface), while the relatively 
low $V p / V s$ ratio of the over-consolidated clayey sequence is favourable to the shear properties inversion.

The medium is parametrised as a stack of 78, $20 \mathrm{~cm}$-thick homogeneous layers, from the seabed at 16 meters below the free surface, down to 15 meters below the seafloor. The MVA velocity profile is used as the starting Vp model. The initial Poisson's ratio model consists of a gradient for the top gravel sequence from 0.5 at the seafloor to 0.45 at the top of the layered sequence, whose value remains constant down to the bottom of the model. The latter is an unusually low value for shallow marine sediments, but is required due to the over-consolidation; tests run with higher average Poisson's ratio resulted in higher data misfit. Although we invert here for the elastic parameters, the forward model includes a long-wavelength $Q p$ model determined using the spectral ratio method from near-offset Boomer and Chirp over the sediment sequence (Pinson et al., 2008); the $Q s$ model is derived from $Q p$ using, in the absence of other information, a high-Q empirical relationship between compressional and shear attenuation, i.e. $Q s=4 / 9 Q p$ (Aki \& Richards, 2002).

\subsection{FWI and comparison with the ground-truth}

In the FWI for the elastic parameters, the strategy described in the methodology section is applied, with a misfit functional based on the complex trace attributes: the modified instantaneous phase is used to obtain a P-wave velocity model robust to cycle-skipping; the elastic model parameters controlling the AVO are then inverted sequentially using the instantaneous trace amplitude. The first stage of the inversion updates the MVA velocity model by fitting the instantaneous phase of the seismogram, starting from a cut-off frequency of $0.5 \mathrm{kHz}$ and progressively broadening the bandwidth up to $1.5 \mathrm{kHz}$. After convergence is attained in the instantaneous phase domain, the envelope of the seismograms are inverted for P-wave velocity; a near-offset taper is applied to the data to attribute higher weight to the near-vertical reflection amplitude and reduce the crosstalk with Poisson's ratio. In Fig. 13, note how the reflection strength inversion updates the velocity model in the absolute values, while the location of peaks and troughs in the velocity structure has already been constrained by the instantaneous phase (IP) inversion. Poisson's ratio inversion 
reduces the misfit between the computed and the field data, with an importance increasing with increasing offset (Fig. 14). No gradient preconditioning is applied in this stage, as the highly heterogeneous impedance in the shallowest two meters would produce a strong bias in the solution, preventing the model from evolving in the deeper portions; nevertheless, the relatively low Poisson's ratio in the site allows for obtaining a stable result. In the final stage, the inversion for density yields minor improvements in the impedance profile; when convergence is reached, density is inverted for independently from P-impedance, alternating steps of Poisson's ratio optimisation (Fig. $15)$.

A synoptic view of the elastic model and the ground-truth is shown in Fig. 16. The seismic inversion site is five hundred meters away from the ground-truth, since the irregular sea bottom at the core site invalidated the $1 \mathrm{~d}$ assumption. However, the lateral continuity of the reflections in the pre-stack-migrated seismic section (Fig. 16) suggests that, other than changes in depths and layer thicknesses, the sediment properties are equivalent. The reflectors of the PSDM seismic section are used to correlate the inversion results to the core measurements, which have been filtered down to the seismic resolution after the removal of outliers and non-significant values. The axis scale of the porosity is reversed, to highlight the positive correlation between the reduction of porosity and the P-impedance (Vardy, 2015); we also expect to find a positive correlation between the clay content and the Poisson's ratio (Hamilton, 1970). The sedimentary log ranges from clay to gravel for loose sediments, whereas the presence of lithified material is marked by values going past the gravel base-line. We divide the section into units, colour coded and labeled in Fig. 16.

Unit a. The top 3 meters of the model are characterised by a high-impedance top portion overlaid by a low-impedance, low-density and lower Poisson's ratio deeper part; although no groundtruth density and porosity are available at this depth, the results are consistent with the gravel layer, with internal heterogeneity confirmed by the presence of reflections in the PSDM section. Note in figure 13 how the inner structure of the gravel layer recognised by the inversion was instead averaged in a uniform high-velocity layer in the MVA model. A strong reduction of the Poisson's ratio is observed from the top to the bottom of the unit, as expected inside the shallowest seabed sediment as a consequence of increasing consolidation with depth. 
Unit b. Here higher impedance and higher Poisson's ratio than the bottom of unit $a$ are obtained; the top and bottom boundaries correlate to two strong reflections in the PSDM section, while the core data identifies a clay bed with a density and porosity structure which mimics the inverted impedance and density profile; note how the strong impedance contrast at the bottom of the unit has no clear correspondence in the density data at the core location, consistently with a weaker and locally less continuous reflection in the PSDM seismic. The increase of Poisson's ratio is in agreement with a more cohesive medium, which is less conducive to shear wave propagation.

Unit c. The impedance and density trends match the porosity and ground-truth density, consistent with changes from fine-grained sediments to silt-dominated beds. Poisson's ratio in this unit is anti-correlated to the impedance profile; this can be explained by an increased stiffness in the micro-fabric of the sediments, which determines an increase of compressional wave velocity correlated to a decrease of the $\mathrm{Vp} / \mathrm{Vs}$ ratio. At the bottom of the unit, the decrease of impedance and increase of Poisson's ratio correlates to a increased clay content in the lithological log.

Unit d. The Impedance and density profiles have a positive gradient, which correlates to the transition to a coarser-grained portion of the lithological log, reaching a maximum in a composite sandy layer interbedded by a thin clay-rich bed; the latter has a distinct low density and high Poisson's ratio signature in the inverted model. The excellent agreement between the ground truth and the model in this layer is encouraging.

Unit e. Impedance and density decrease in a one-meter thick bed interbedded by a higher impedance and density thinner layer. This correlates with a clay bed interbedded by a thin coarsergrained interval. Poisson's ratio shows a decrease in this unit, with a negative peak corresponding to the coarse-grained layer depth and an increase in the clay content towards the base. Density and porosity from the $\log$ are consistent with the inversion results.

Unit f. High impedance and density unit, interbedded by a $20 \mathrm{~cm}$ thick bed with low impedance and density. The ground-truth shows a similar pattern and correlates the low-impedance layer with a shelly sediment bed within the clay-dominated sediment. Poisson's ratio has a negative trend at the top, reaches a negative peak at the low-impedance sand layer location, and sharply increases 
in the bottom clay-rich portion. In this unit, changes between cohesive and granular medium have a clear signature on the Poisson's ratio variations.

Unit g. This unit shows an alternation of lows and highs in impedance and density. The sharp increase of impedance at the bottom corresponds to a strong continuous reflection in the PSDM seismic; this correlates to a fining-upward, from gravel to clay, sediment interval. The internal lithological layering seems to have a signature in the $20 \mathrm{~cm}$ thick sharp decrease of Poisson's ratio at a depth compatible with the gravely-sandy part. Note how, at this depth, the quality of the inverted density profile has deteriorated as a consequence of the narrower reflection angle range.

Unit $\mathbf{h}$. The top part of this unit shows a decrease of impedance and density, which correlates to the density and porosity profiles, followed by an increase which can be related to the top of a rocky unit.

\subsection{Comments}

The real data example corroborated most of the conclusions drawn from the synthetic sensitivity tests. The combination of streamer aperture and water depth ensured that the reflection angle range was wide enough to obtain an independent characterisation of the subsurface in terms of P-wave velocity, density and Poisson's ratio, without assuming any a-priori relationship between the parameters. Despite the simple starting model used and the deterministic approach, a sensible solution has been obtained. The convergence properties of the modified instantaneous phase have been exploited to reduce the risk of cycle-skipping, whereas possible inaccuracies in the source wavelet have been mitigated by using the reference-trace normalisation. The offset-dependent misfit evolution throughout the inversion confirmed that the sequential optimisation is a reasonable and effective way to tackle the hierarchical dependancy of the data on the elastic parameters. The presence of strong internal heterogeneity inside the top gravel layer undermined the effectiveness of the Poisson's ratio gradient preconditioning scheme; however, the relatively low Poisson's ratio in the site and the wide reflection angle, allowed for the shear properties inversion to produce stable results using a raw gradient calculation. 
G. Provenzano et al.

The $Q p$ macro-model estimated using the spectral ratio method (Pinson et al., 2008) and the derived $Q s$ were included in the forward model, in particular to account for the strong intrinsic attenuation within the top low- $Q p$ gravel layer. Although a high-fidelity attenuation model would further improve the characterisation of the sediment column, a reliable and precise wavelengthscale $Q p$-model is difficult to achieve: in the high $Q p$ limit there is little attenuation per wavelength, and in the low $Q p$ limit the energy is unable to propagate. In particular, within the FWI framework, there is low sensitivity to attenuation and strong crosstalk with the elastic model parameters (e.g. Malinkowski et al., 2011; Kamei \& Pratt, 2013); furthermore, constraining Qs from marine seismic data would require isolating converted S-waves. In this case study, the sensitivity to $Q$ is further limited by the large-scale high quality factor of the fine-grained, cohesive sediments that form the dipping beds (Pinson et al., 2008; Malinkowski et al., 2011), which limits the change in waveform due to attenuation. However, the large-scale, high precision, $Q p$ model of Pinson et al. (2008) accounts for the intrinsic attenuation of energy, enhancing the stability of the inversion and the reliability of the solution.

\section{CONCLUSIONS}

This work demonstrates that it is possible to use a multi-channel UHF sub-bottom profiler to derive a high-fidelity distribution of the elastic properties of the sub-seabed with a sub-metric resolution, using a relatively simple algorithm, with a simple pre-processing of the raw data and without detailed a-priori information. A dedicated modelling and inversion strategy has been developed to account for the specific acquisition conditions and frequency content of shallow marine data within a reasonable computing time on a standard workstation. A broad range of synthetic tests along with a real data case study have been used to explore the capabilities of the inversion method and to define the conditions under which a full elastic characterisation can be obtained.

We have shown that:

- A sequential inversion strategy is an efficient way to tackle the multi-parameter dependancy of the data, and can be used to derive P-wave velocity, bulk density, and Poisson's ratio profiles. 
- The relative contribution of density and P-wave velocity to P-impedance can be decomposed, provided that reflection angles $>40$ degrees are available.

- P-impedance is the most robust parameter to noise and can be effectively obtained from narrow reflection angle data even if P-wave velocity and density cannot be separated, as long as a reliable low-wavenumber velocity model is available.

- The Poisson's ratio inversion does not require wide reflection angle data, but it suffers from an important loss of sensitivity in very high $\mathrm{Vp} / \mathrm{Vs}$ ratio media, which can be partially made up for using a structure-oriented gradient preconditioning.

The elastic model obtained shows a good agreement with the ground-truth density and porosity, and a correlation is shown between clay content and Poisson's ratio distribution. Estimates of physical properties, such as undrained shear strength, effective stress, overpressure ratio and porosity, can be derived from the inverted elastic model (Hamilton, 1970; Richardson \& Briggs, 1993; Mavko et al., 2009; Vardy, 2015). This opens the way to the extensive use of quantitative seismic interpretation as a means to obtain a detailed characterisation of the shallow sediment properties, as an efficient alternative to costly and time-consuming coring campaign.

\section{ACKNOWLEDGMENTS}

The authors wish to thank Luke Pinson and John Davis for their help in acquiring the data used as the field data case study, along with Tim Minshull and Antonis Zervos for their informative discussions regarding inversion and geomechanics. We also thank Ludovic Métivier, Richard Hobbs and an anonymous reviewer for the useful comments, that greatly improved the quality of the manuscript. The field data preconditioning was performed using a combination of Landmark's ProMAX software, MatLab, and Seismic Unix. The full waveform forward modelling was performed using the MIT OASES algorithm. All other processing used custom-written algorithms. 


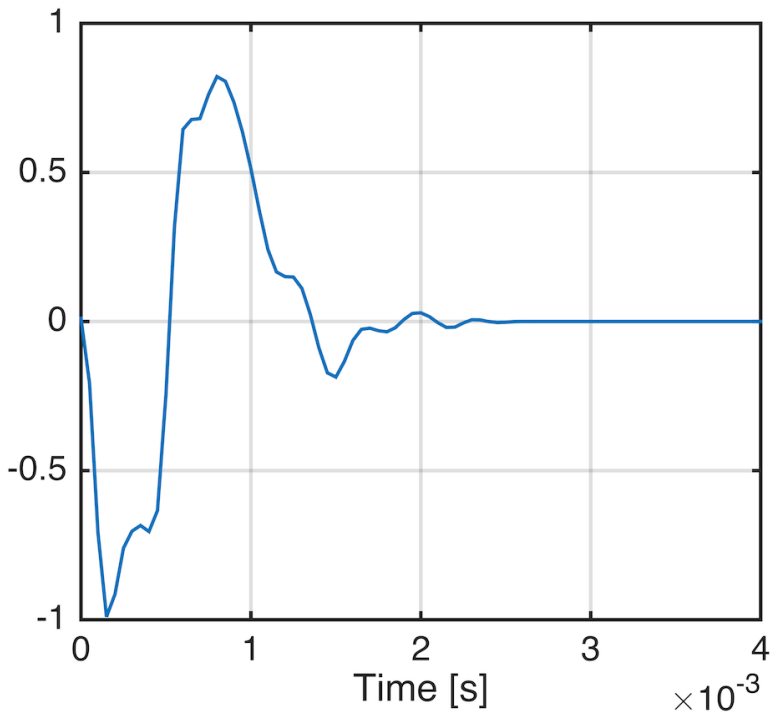

(a)

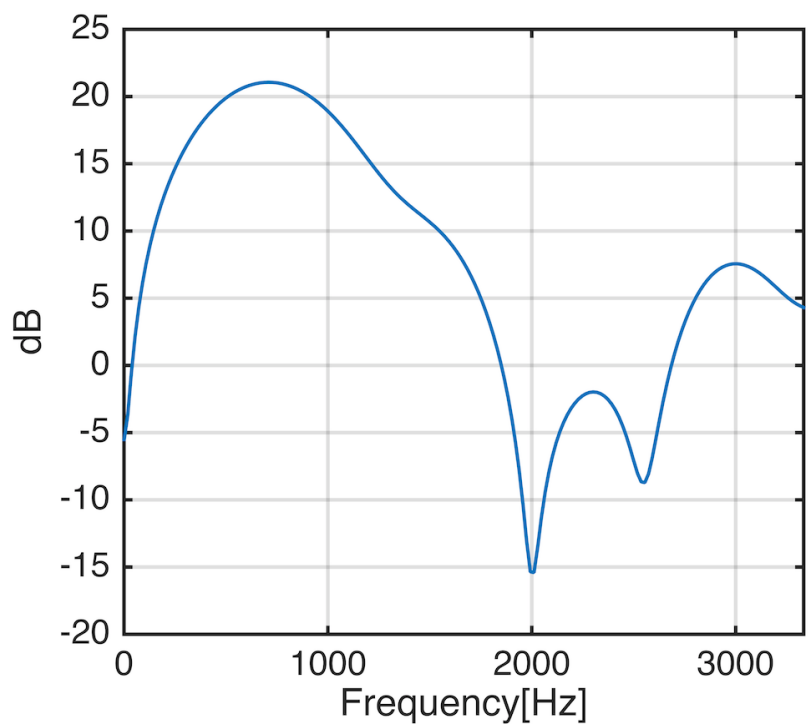

(b)

Figure 1. Boomer source wavelet. a) Time domain source signature. b) Power spectrum in deciBel 

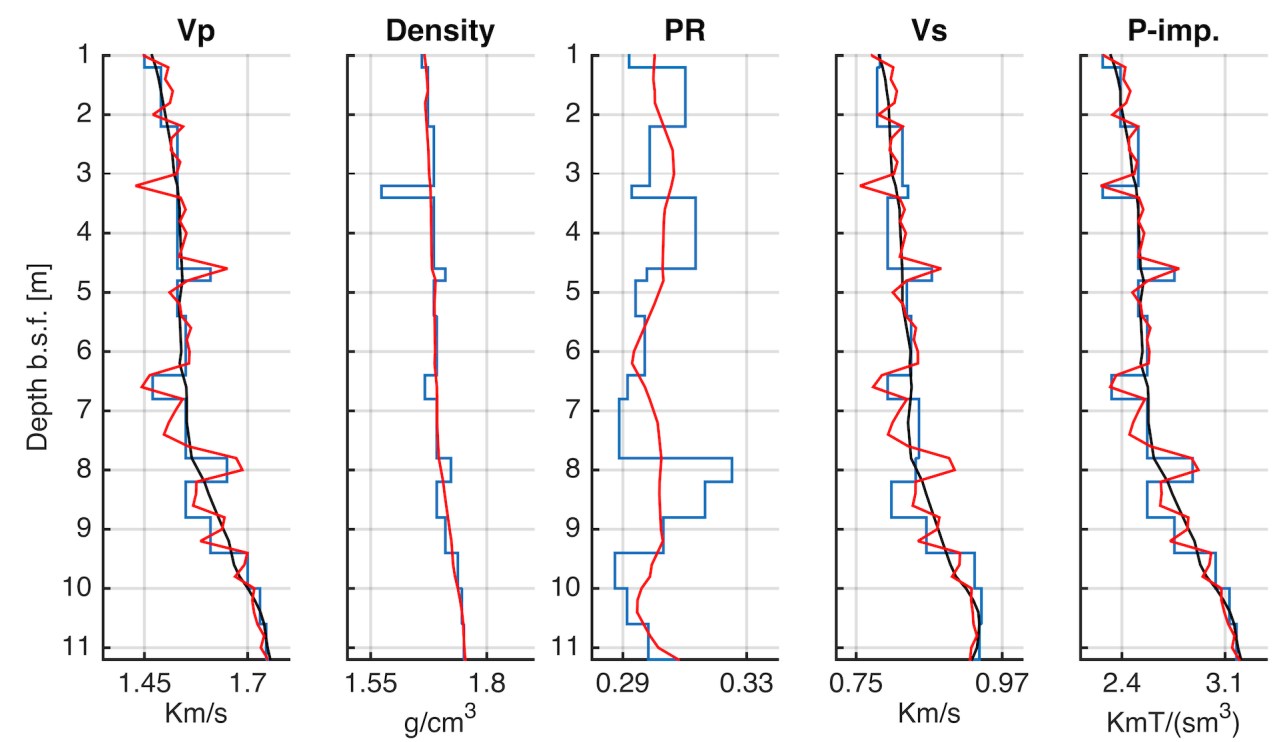

(a)

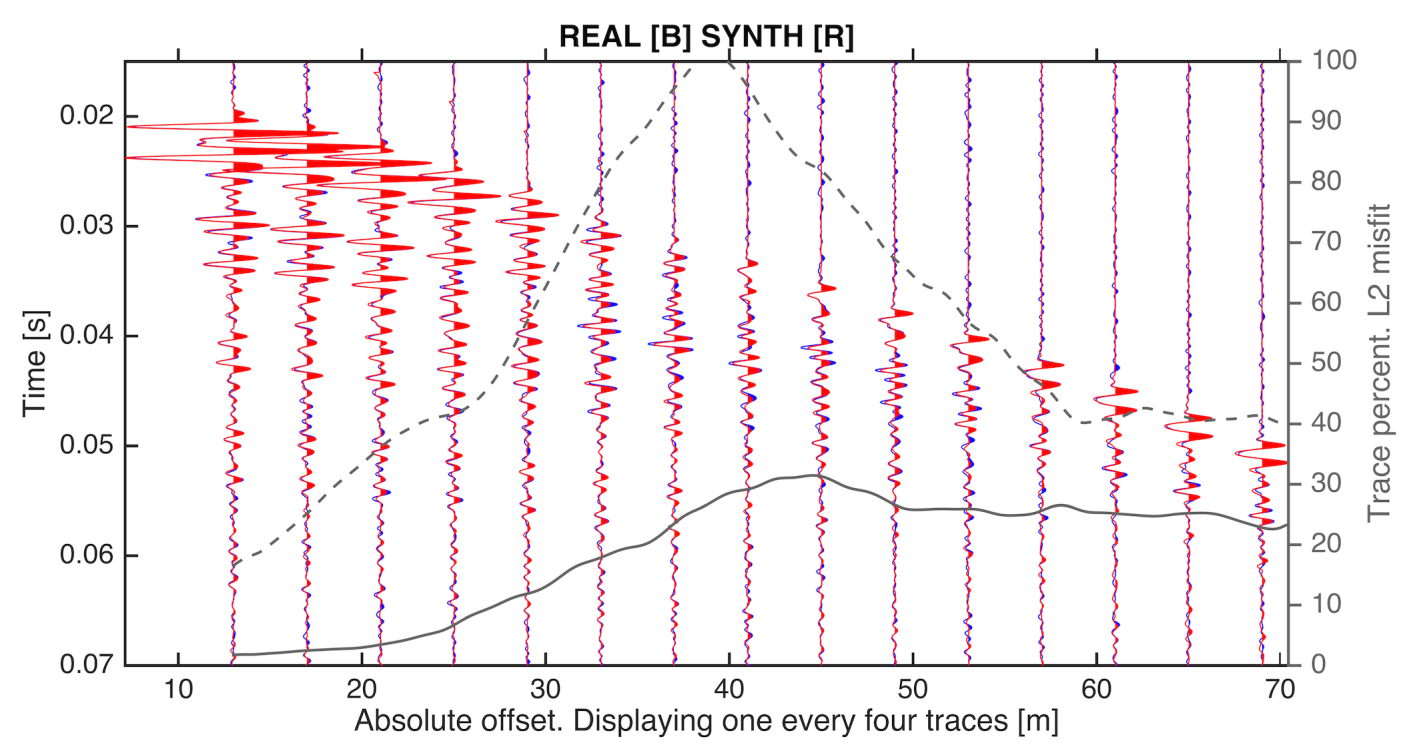

(b)

Figure 2. Stage1, P-wave velocity inversion. a) Starting model (black), true model (blue), current best model (red). b) Synthetic seismogram (red) overlaid to the "real data" (blue). The solid grey curve represents the trace by trace percentage L2 misfit for the final model, the dashed curve is relative to the model at the beginning of the stage. The Impedance profile is retrieved accurately and a good match is attained in the short offsets of the seismic gather. 

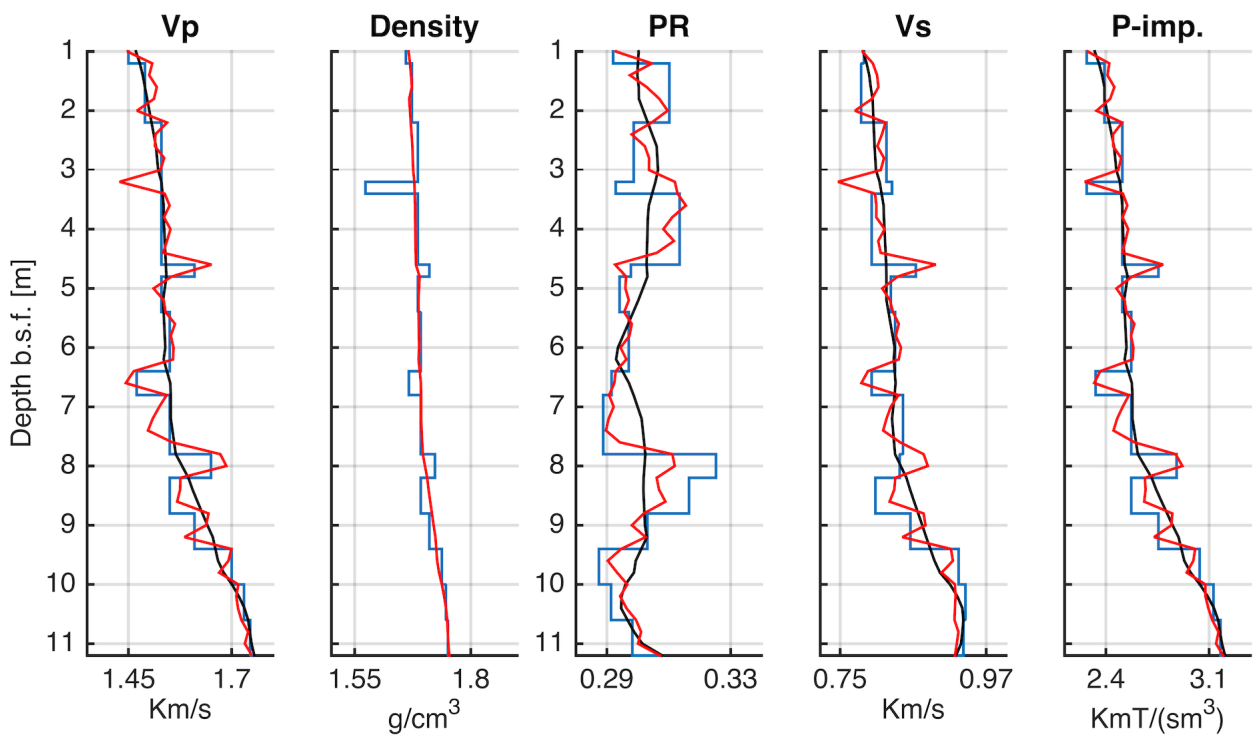

(a)

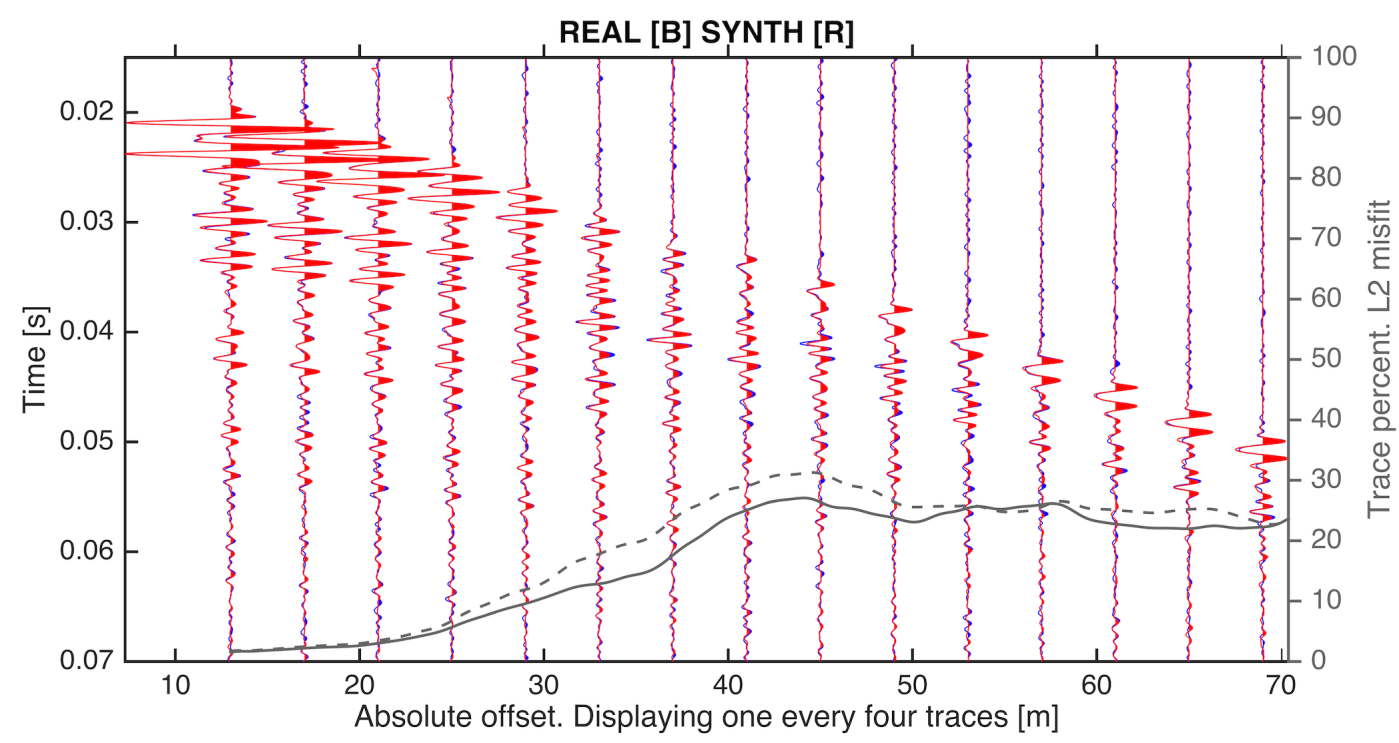

(b)

Figure 3. Stage2, Poisson's ratio inversion. a) Starting model (black), true model (blue), current best model (red). b) Synthetic seismogram (red) overlaid to the "real data" (blue). The solid grey curve represents the trace by trace percentage L2 misfit for the final model, the dashed curve is relative to the model at the beginning of the stage. In this stage we reduce the data misfit associated to the AVO characteristics of the gather, but the inaccuracies in the starting density model prevent the algorithm from retrieving the shearproperties' fine-scale details. 

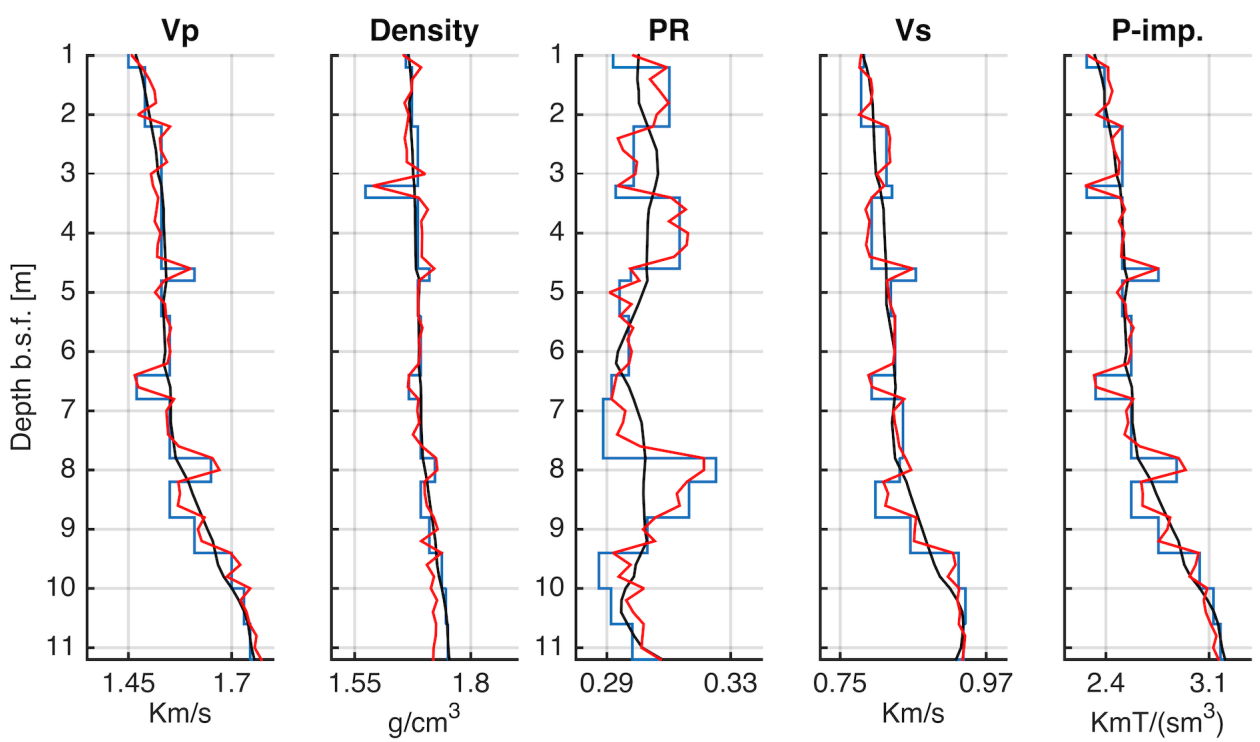

(a)

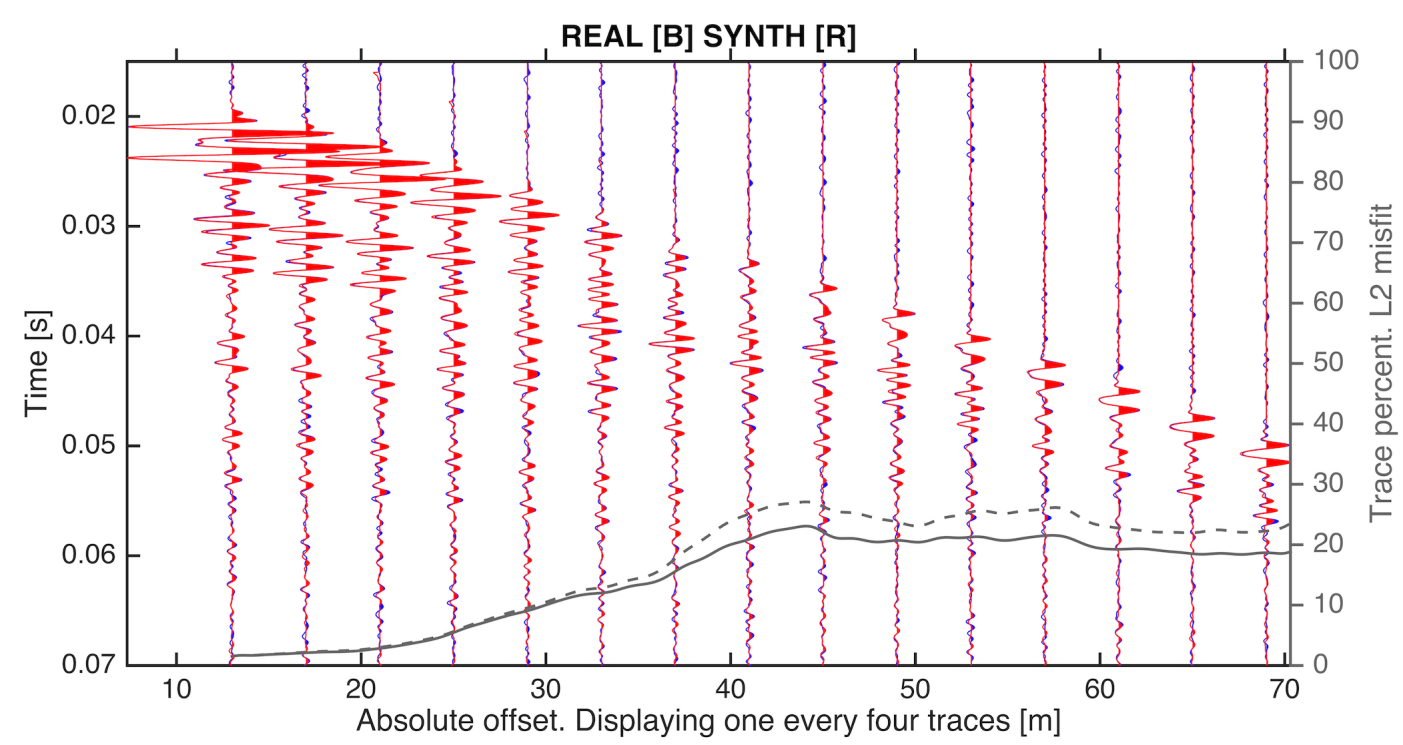

(b)

Figure 4. Stage3, Cyclical Density and Poisson's ratio inversion. P-impedance constant. a) Starting model (black), true model (blue), current best model (red). b) Synthetic seismogram (red) overlaid to the "real data" (blue). The solid grey curve represents the trace by trace percentage L2 misfit for the final model, the dashed curve is relative to the model at the beginning of the stage. In this stage we fit the wide-angle part of the shot gather by optimising cyclically for Density and Poisson's ratio. Note how the impedance change right below 3 meters depth is correctly attributed to a density change and the P-wave velocity is changed accordingly to keep the P-impedance constant. 


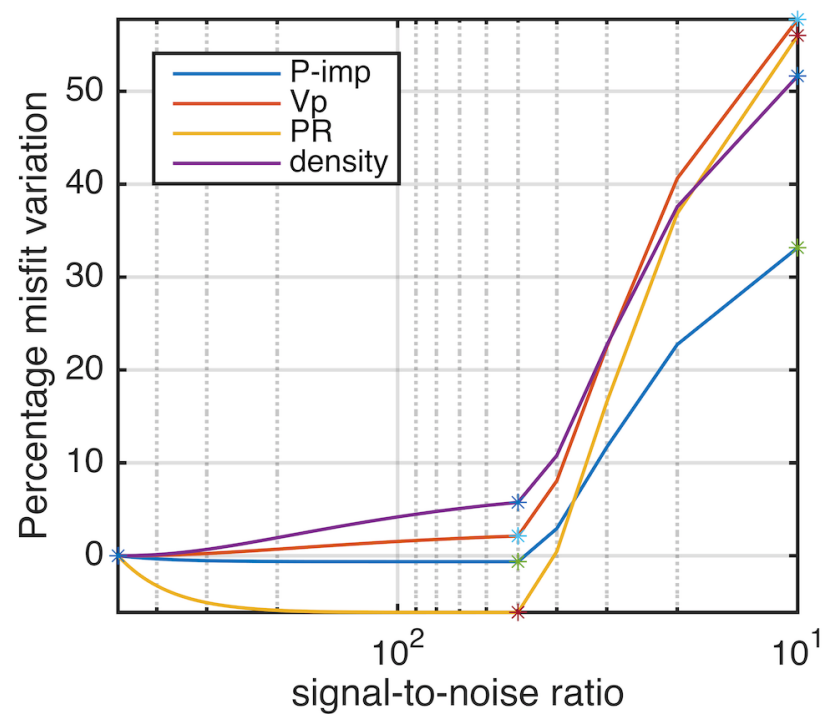

Figure 5. Robustness to random noise. Model misfit percentage variation for P-impedance, Poisson's ratio, $\mathrm{P}$-wave velocity and density as a function of signal-to-noise ratio (SNR). Note how the acoustic impedance is the most robust parameter, while the $\mathrm{Vp} /$ density separation is the most sensitive to the noise energy in a broad SNR range. Interpolated from the computed value (asterisks). 


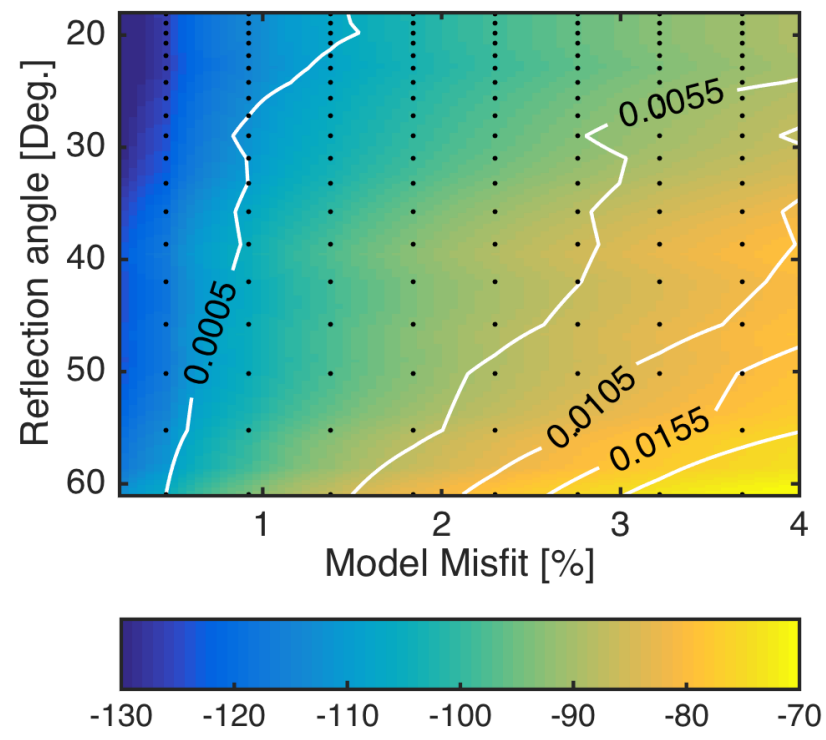

(a)

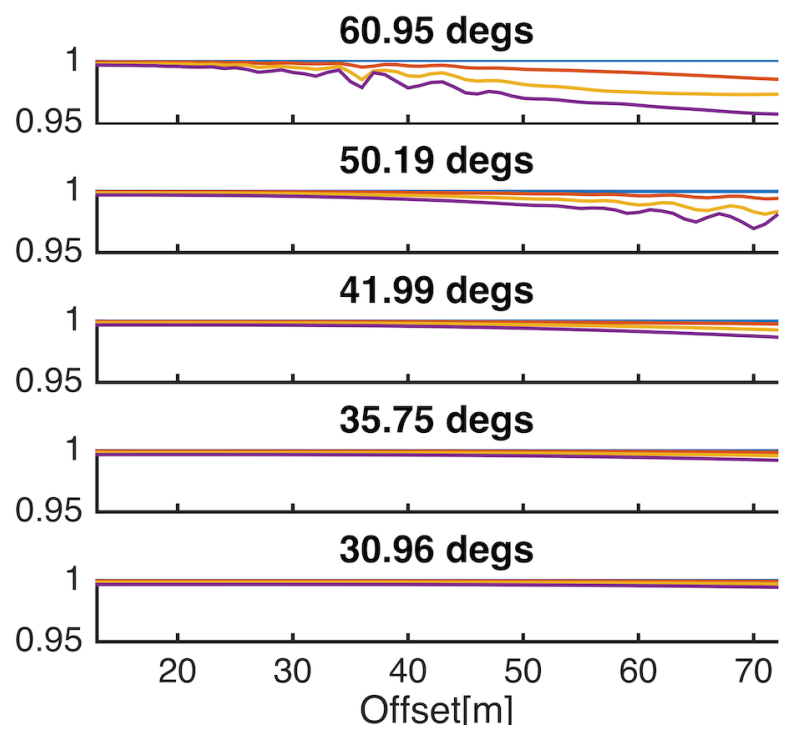

(b)

Figure 6. Constant-impedance density sensitivity analysis. Per each reflection angle, the $L 2$ data misfit normalised to the seismogram energy is computed between a reference model and a range of models with increasing percentage perturbation of the target layer density. a) Data misfit normalised to the total data energy in deciBel. The contour plot shows the percentage iso-misfit surface. The black dots correspond to the computed models. b) Maximum cross-correlation value as a function of offset at different maximum reflection angles. The offset-dependent correlation is computed per different perturbation values (increasing from pale blue to magenta). 


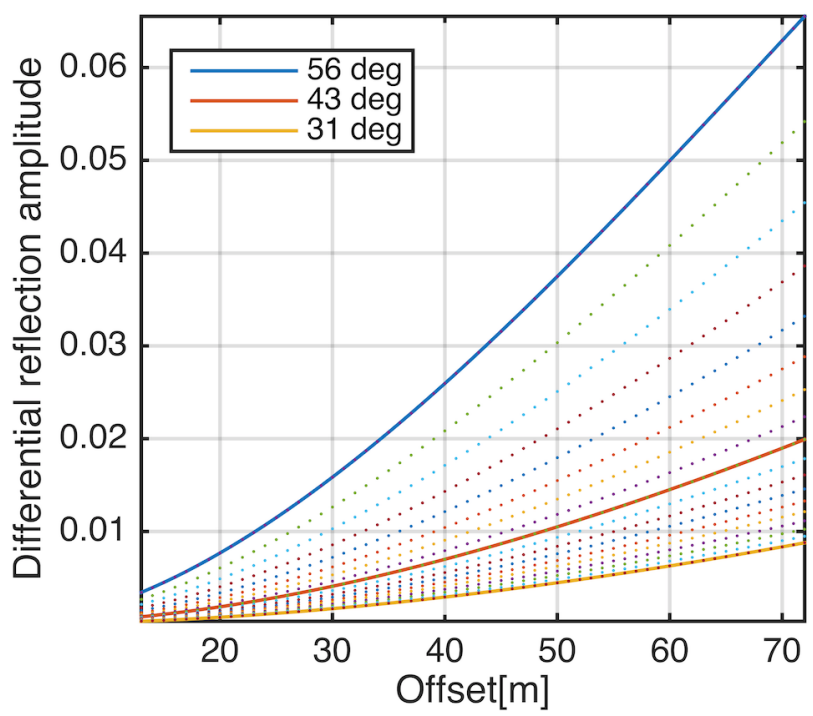

(a)

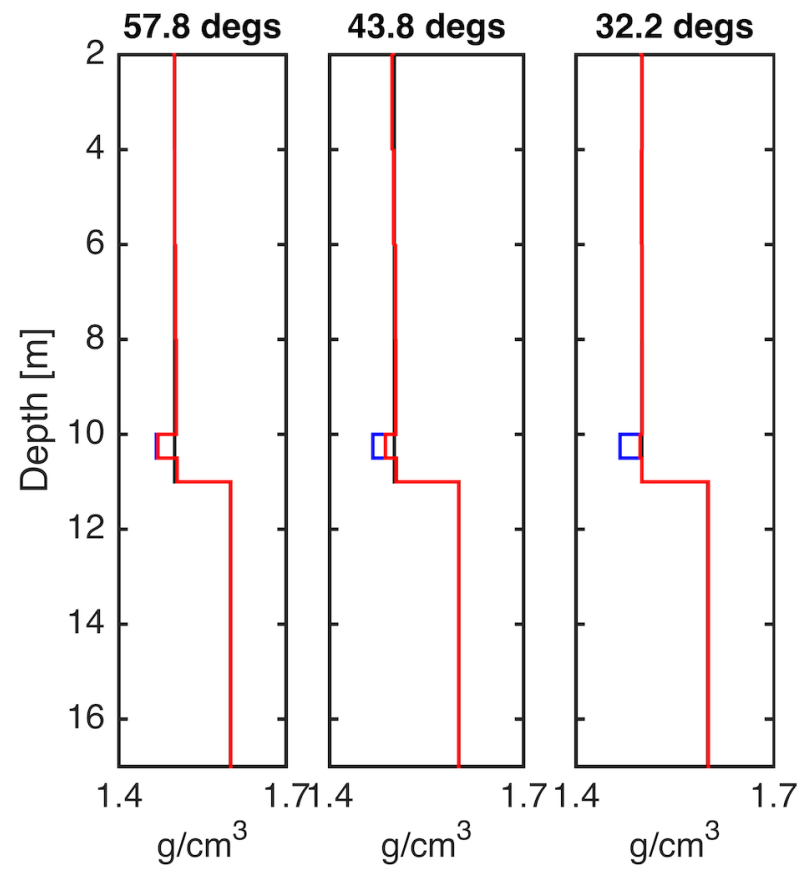

(b)

Figure 7. Constant-impedance density sensitivity analysis. a) Differential reflection AVO computed between a constant density model, and a model where both density and Vp contribution are present, within a reflection angle range corresponding to changes in sea-floor depth from 15 to 50 meters. The solid lines are relative to the models considered for the inversion. The legend contains the reflection angle at the target layer. b) Retrieved density profile (red), against true density profile (blue). The starting density model is homogeneous (black), although it corresponds to a correct P-impedance profile. 


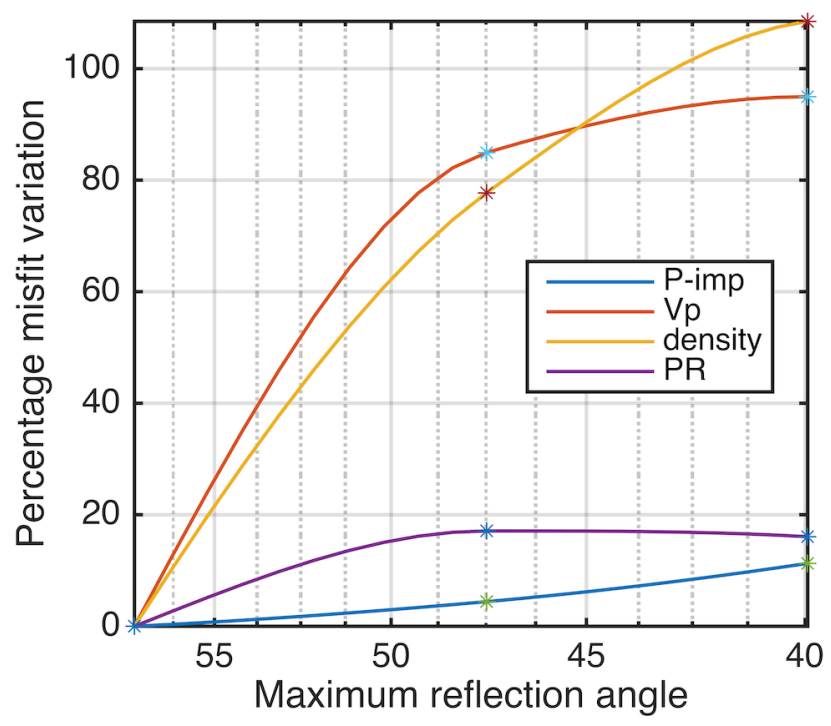

Figure 8. Robustness to changes in the reflection angle range. Model misfit percentage variation as a function of the maximum reflection angle for the complex 18 layers model per each parameter class. Interpolated from the computed value (asterisks). 


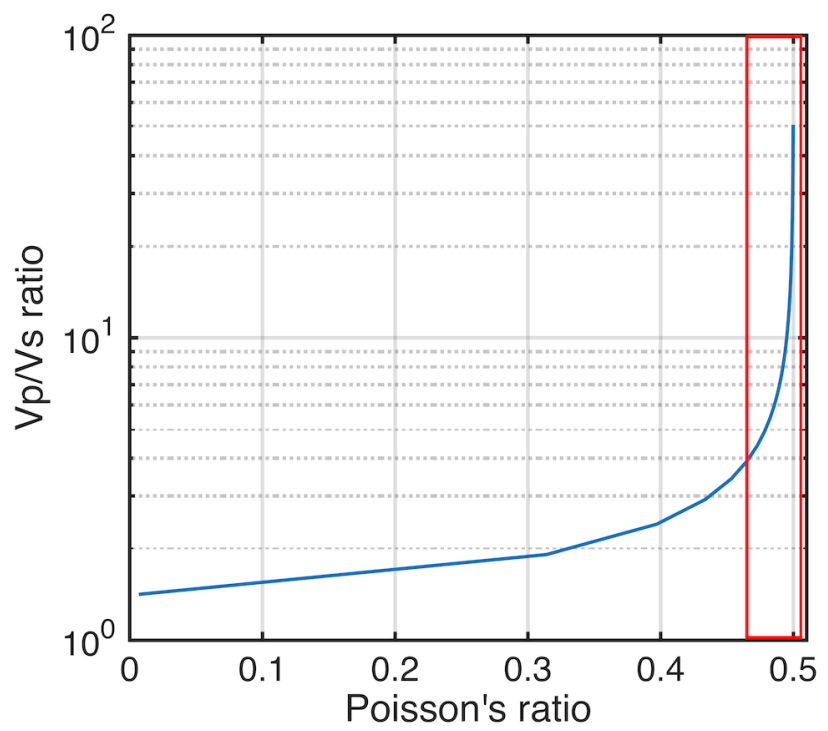

(a)
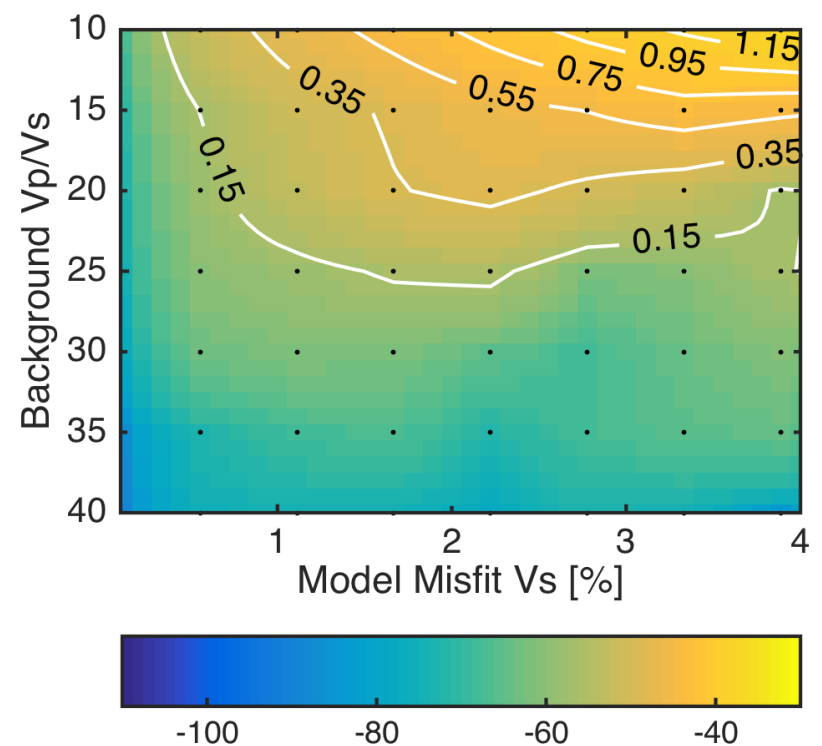

(b)

Figure 9. Poisson's ratio sensitivity. a) Poisson's ratio vs Vp/Vs ratio. Most unconsolidated sediments fall into the red box, where great changes in $\mathrm{Vp} / \mathrm{Vs}$ correspond to a narrow Poisson's ratio range and thereby to a small AVO effect, which translates into a poorer sensitivity. b) Over a broad $\mathrm{Vp} / \mathrm{Vs}$ ratio range of the encasing medium, the L2 data misfit is computed between a reference model and a range of models with increasing percentage perturbation of the target layer S-wave velocity. Data misfit normalised to the total data energy in deciBel. The contour plot shows the percentage iso-misfit surface. The black dots correspond to the computed models. 


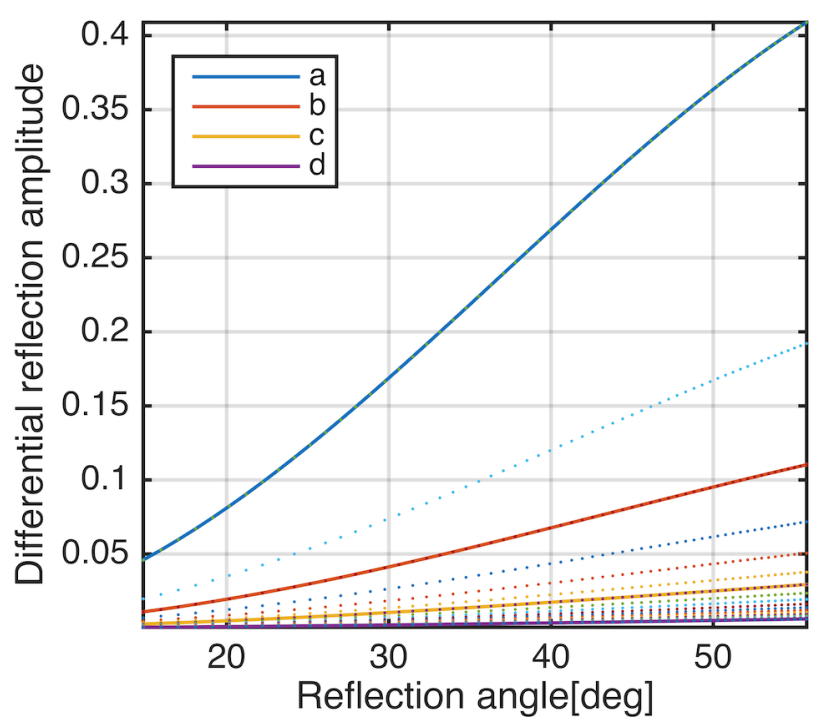

Figure 10. Poisson's ratio sensitivity. Difference between the AVA of the starting and true Poisson's ratio profiles in the four $\mathrm{Vp} / \mathrm{Vs}$ ranges considered in the sensitivity analysis (solid lines). Intermediate differential AVA curves are shown with the dotted lines. The quick fall in differential AVA from the first solid line $(\mathrm{Vp} / \mathrm{Vs}=2)$ to the first dotted line $(\mathrm{Vp} / \mathrm{Vs}=3)$ is consistent with the quick loss in sensitivity shown in figure $9 \mathrm{~b}$ 


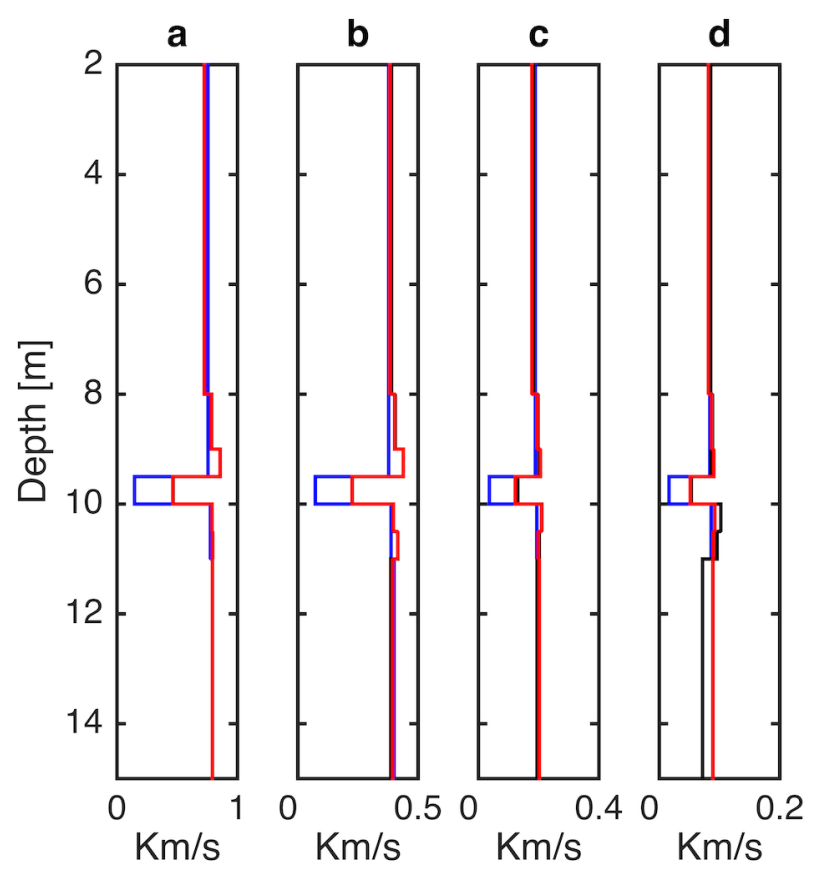

(a)

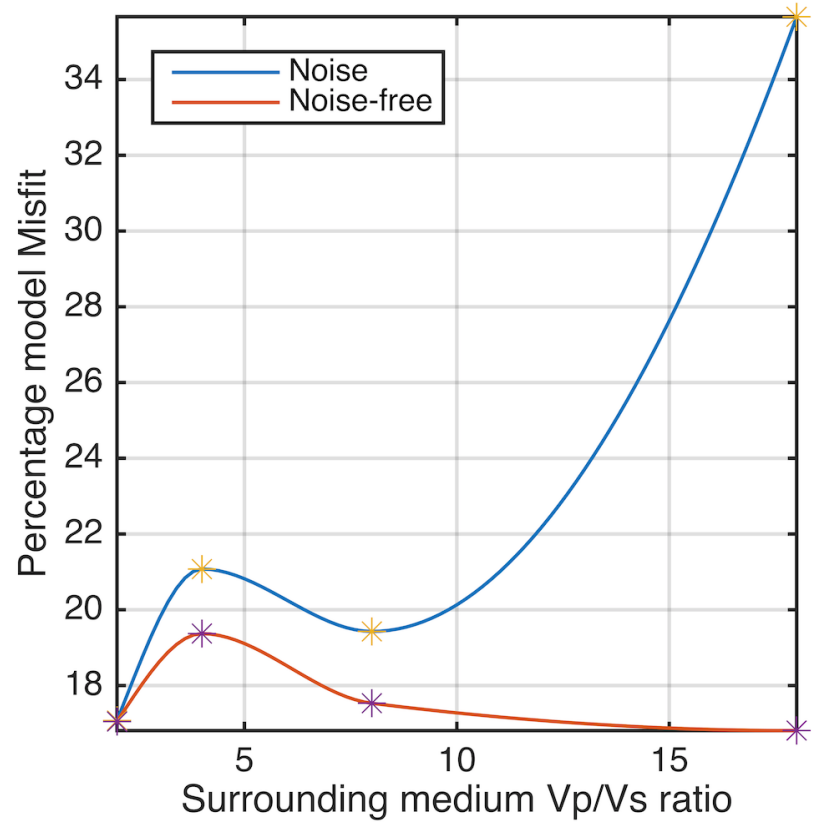

(b)

Figure 11. Poisson's ratio inversion as a proxy to Vs. Sensitivity analysis. Poisson's ratio inversion for the models from $a$ to $d$, with and without noise. a) Retrieved S-wave velocity profile in the noise-free (red) and noise-contaminated data (black), against the true Vs profile (blue). b) Final Vp/Vs ratio misfit for models from $a$ to $d$ in the noise-free and noise-contaminated cases. Interpolated from measured data (asterisks). 


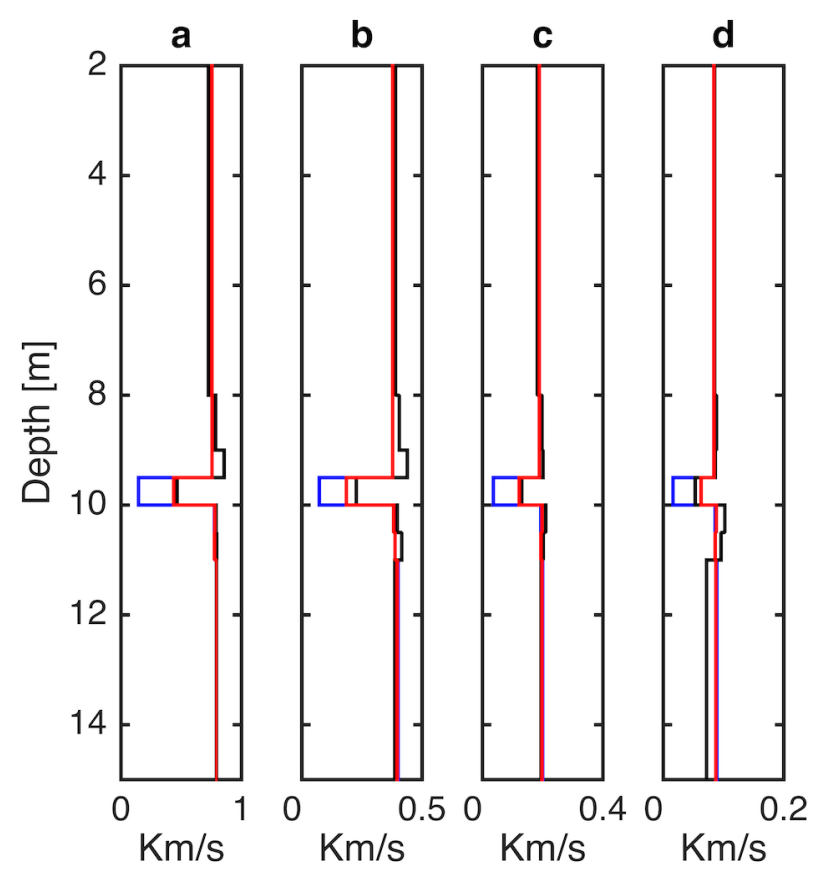

(a)

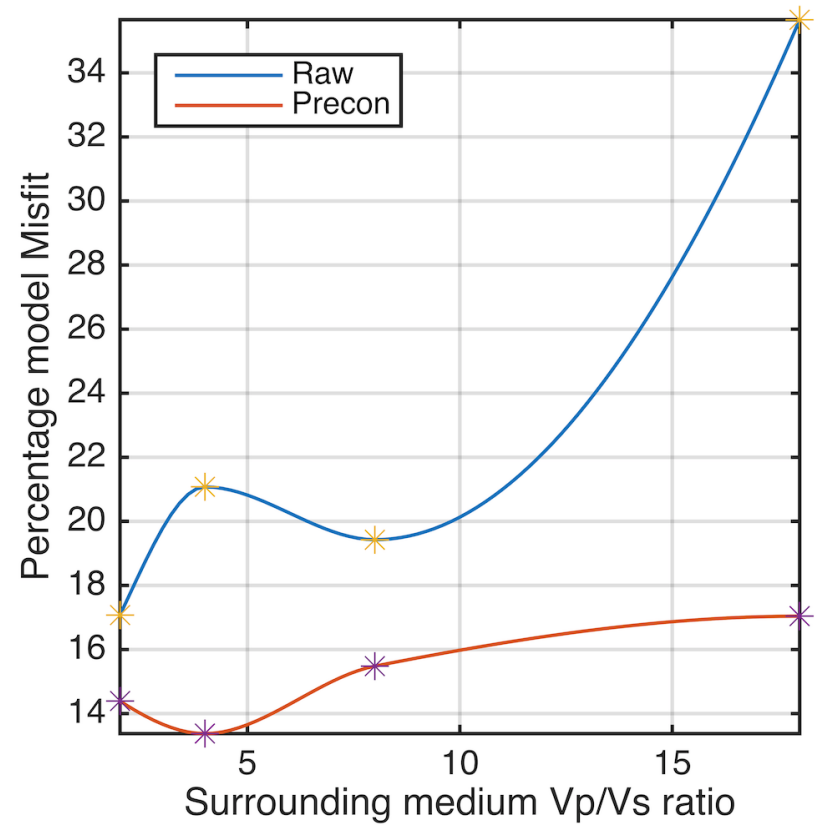

(b)

Figure 12. Poisson's ratio inversion as a proxy to Vs. Sensitivity analysis. Poisson's ratio inversion of models from $a$ to $d$ with structure-constrained gradient preconditioning. Noise-contaminated data. a) Retrieved S-wave velocity profile with (red) and without (black) gradient preconditioning, against the true Vs profile (blue). b) Final Vp/Vs ratio misfit for models from a to $\mathrm{d}$ with and without gradient preconditioning. Interpolated from measured data (asterisks). 

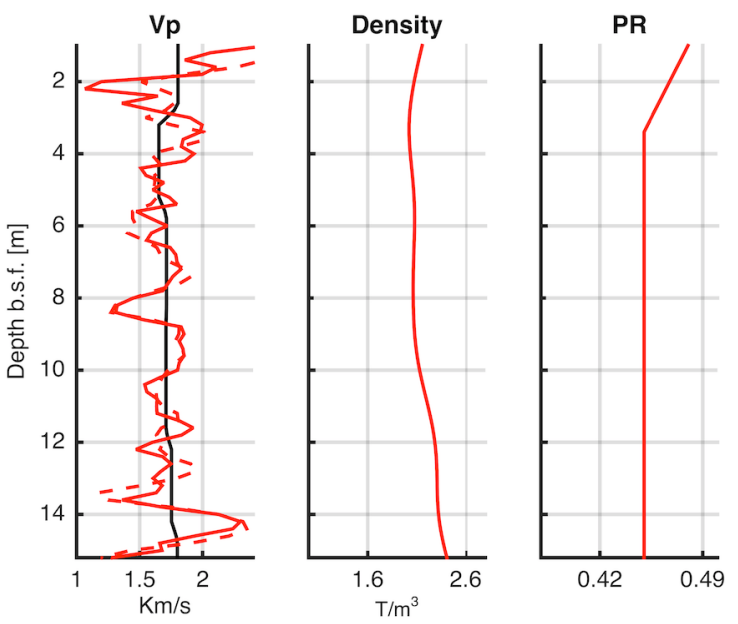

(a)

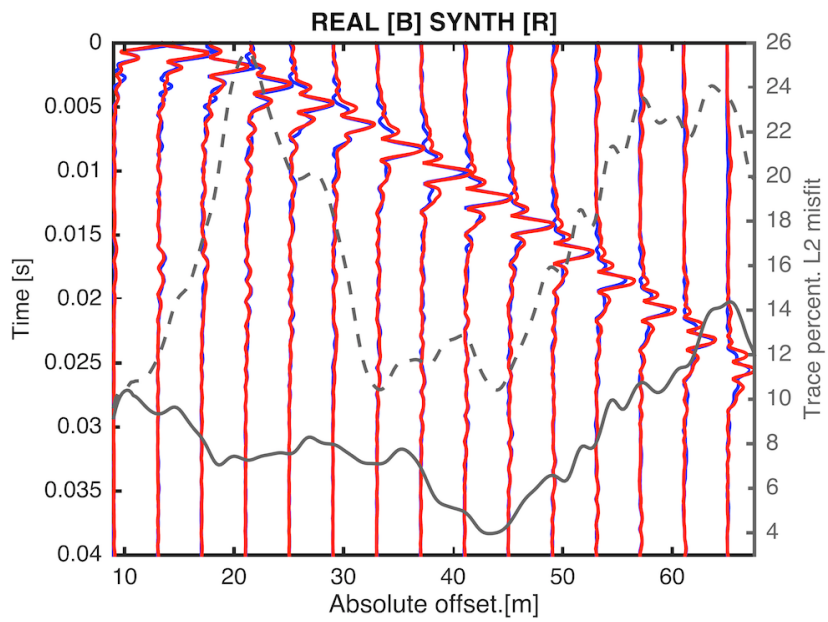

(b)

Figure 13. Stage1. P-wave velocity inversion. Instantaneous phase multi-scale P-wave velocity inversion, followed by a P-wave velocity instantaneous amplitude inversion with short offset windowing. In panel a, the starting model (black) and the final model at this stage (red). The dashed red line is the Vp model after the instantaneous phase inversion only. In panel $b$, the envelope of the synthetic seismogram (red) overlaid to the real (blue) and percentage misfit as a function of offset, at the start and at the end of the stage (dashed and solid grey lines). 

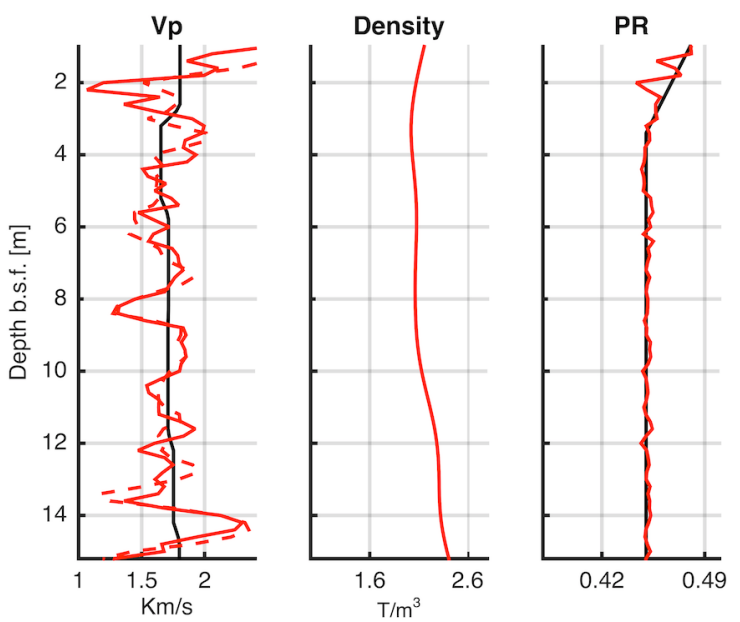

(a)

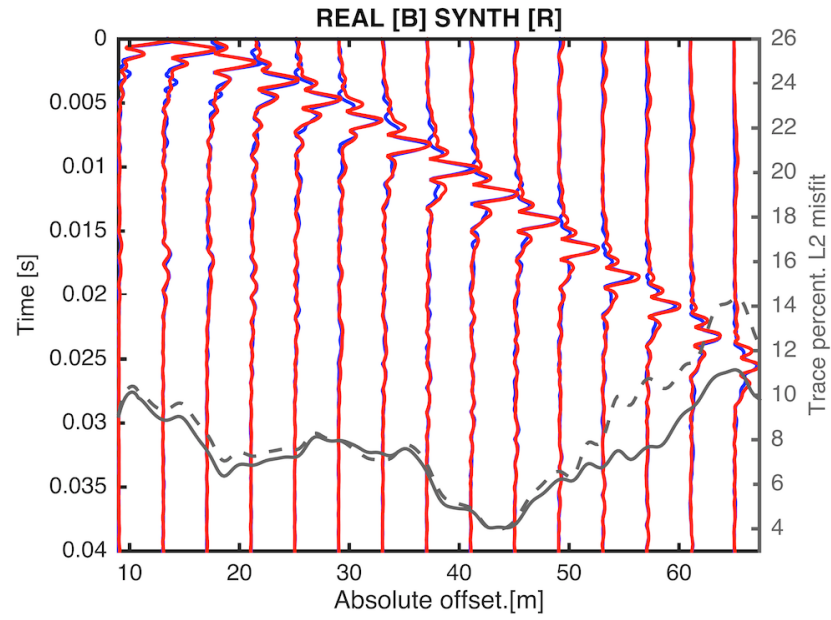

(b)

Figure 14. Stage2. Instantaneous amplitude Poisson's ratio inversion. In panel a, the starting model (black) and the final model at this stage (red). In panel b, the envelope of the synthetic seismogram (red) overlaid to the real (blue) and percentage misfit as a function of offset, at the start and at the end of the stage (dashed and solid grey lines). 

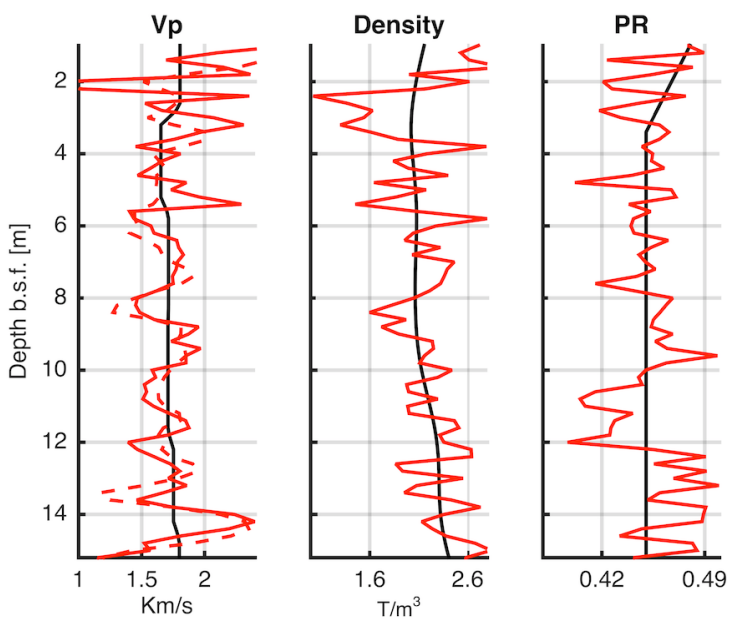

(a)

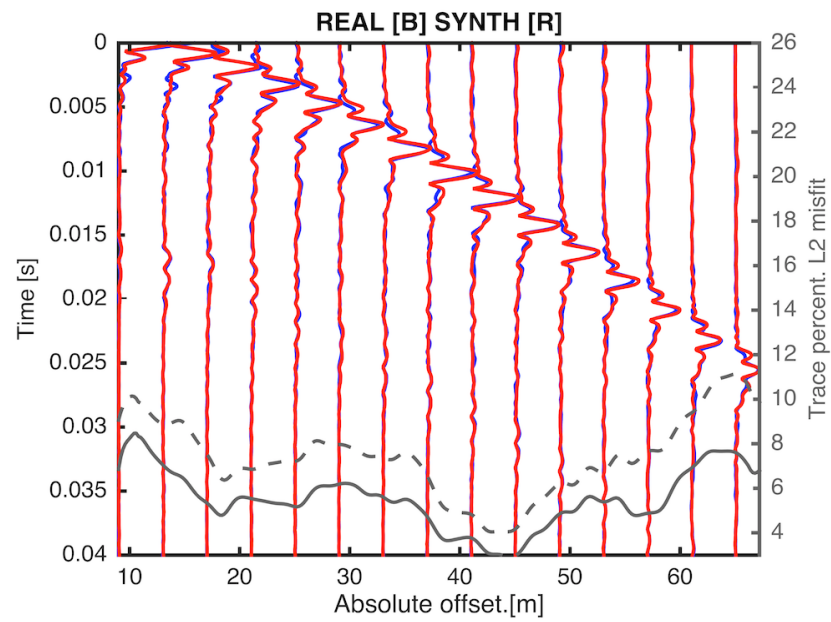

(b)

Figure 15. Stage 3. Instantaneous amplitude cyclical Density and Poisson's ratio inversion. Firstly, density is updated independently from $\mathrm{Vp}$ to optimise the impedance; after convergence, impedance is kept constant and density and Poisson's ratio are inverted for cyclically. In panel a, the starting model (black) and the final model at this stage (red). In panel b, the envelope of the synthetic seismogram (red) overlaid to the real (blue) and percentage misfit as a function of offset, at the start and at the end of the stage (dashed and solid grey lines). 

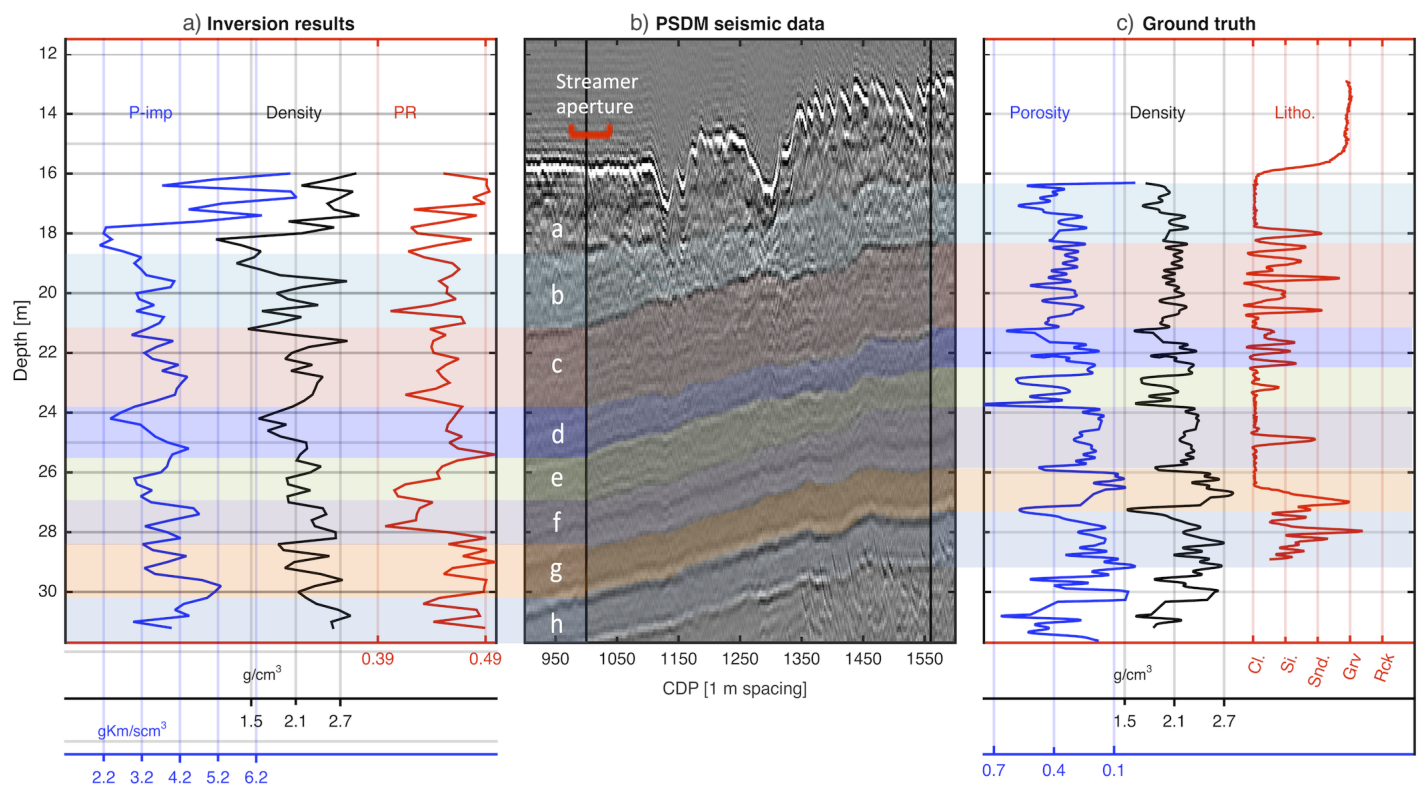

Figure 16. Inversion results interpretation. In panel a) inverted P-impedance, density and Poisson's ratio. In panel b) PSDM seismic section with location of the acquisition site (left vertical line) and core position (right vertical line). Overlaid to the acquisition site, the red line indicates the width of the acquisition streamer. Note how the vertical dimension is greatly exaggerated. In panel c) wet bulk density, fractional porosity and sedimentary column from log; the latter is a curve whose values are proportional to the relative abundances of clay, silt, sand, gravel and lithified sediment. The labelled units are colour-coded to highlight the correlation between the inversion results and the ground-truth. 


\section{APPENDIX A: EFFICIENT RECEIVER GHOST PREDICTION IN THE GENERAL VARYING STREAMER DEPTH CASE}

A recorded seismic reflection pressure wavefield is the convolution between a purely up-going wavefield and the impulse response of the acquisition system as a function of receiver depth, freesurface reflection coefficient and spatial frequency (Aytun, 1999). The total wavefield at depth $h$ in the frequency-wavenumber domain can be obtained from the up-going field $\mathbf{P}_{k}{ }^{-}\left(h_{0}\right)$ as:

$\mathbf{P}_{k}\left(h, r_{0}\right)=\mathbf{P}_{k}{ }^{-}\left(h_{0}\right) \mathbf{F} 1_{k}\left(h, r_{0}\right) \mathbf{F} 2_{k}\left(h, h_{0}\right)$

where:

$\mathbf{F} 1_{k}\left(h, r_{0}\right)=\left(1+r_{0} e^{\left(-j 2 k_{z} h\right)}\right)$

predicts the receiver ghost at depth $h$ as a function of the vertical wavenumber $k_{z}=\sqrt{k^{2}-k_{x}^{2}}$, with a free-surface reflection coefficient equal to $r_{0}$, whereas:

$\mathbf{F} 2\left(h, h_{0}\right)=e^{\left(j k_{z}\left(h-h_{0}\right)\right)}$

is the downward propagation operator from $h_{0}$ to $h$ accounting for the travel-time difference per each plane-wave component. The time-offset domain seismogram is obtained via two-dimensional Fourier inverse transformation:

$\mathbf{d}(t, x)=\mathcal{F}^{(-1)}\left\{\mathbf{P}_{k}\left(h, r_{0}\right)\right\}$

In the general case of offset-varying streamer depth and sea-surface reflection coefficient, the complete $N$-channels seismic gather can be obtained by selecting each trace from the corresponding redundant-offset domain gather and then merging the offsets. A compact mathematical formulation exploits the properties of the two-dimensional dirac delta function:

$\mathbf{d}_{i}(t, x)=\mathcal{F}^{(-1)}\left\{\mathbf{P}_{k}\left(h(i), r_{0}(i)\right)\right\}$
$\mathbf{d}(t, x)=\sum_{i=1}^{N} \int_{-\infty}^{+\infty} \int_{-\infty}^{+\infty} \delta\left(t-\tau, x_{i}\right) \mathbf{d}_{i}\left(\tau, x_{i}\right) d \tau d x$

As opposed to an explicit full wavefield modelling, only the computation of the up-going wavefield at constant depth is required, and the whole gather is obtained by signal manipulation, with significant savings in the computing cost. 
In general, the filter parameters $h$ and $r_{0}$ are not known in advance with the accuracy required by UHF seismic modelling and need to be estimated from the data. The receiver ghost loci in the frequency-offset $(\mathrm{Fx})$ domain are a function of the receiver depth:

$f n_{\left(x_{i}\right)}=n \frac{V_{w}}{2 h_{x_{i}} \cos (\theta(i))}$

where $n$ indicates the order of the harmonic, $V_{w}$ is the water layer velocity and $\theta$ is the reflection angle from the sea-floor, which can be approximated as the ratio between the traveltimes (Pinson, 2009):

$\theta=\left(t_{0} / t_{i}\right)$

provided that $t_{0}$ corresponds to a near-vertical reflection. Eq. A.7 establishes a linear relationship between receiver-ghost null-frequencies and the reciprocal of the receiver depth as a function of offset. The estimation of the offset-dependent receiver depths can be then cast as a linear inverse problem (Aster et al., 2005); provided that more than one harmonic per offset is available, the problem is over-determined and the linear formulation is convenient to derive robust confidence intervals for the solution (Menke, 1989; Aster et al., 2005). In Fig. A1 we show the observed null frequency versus the predicted null frequency for the estimated depth, overlaid to the frequencyoffset amplitude spectrum of the real data. The estimated depth profile in the lower panel shows a clearly sagging geometry and the effect of the tail-buoy in the far-offset channels.

The reflection coefficient per each each channel can be conveniently obtained as the solution of a non linear optimisation problem using deterministic algorithms as simple as the bisection method (Burden \& Faires, 1985); the optimal $r_{0}\left(x_{i}\right)$ maximises the normalised cross-correlation value between the predicted trace and the desired trace at the appropriate offset. Although this step is model-based, it only requires an accurate a-priori estimate of the sea-floor depth: the normalised cross-correlation removes the dependency of the results on the accuracy of the sea-floor reflection AVO, while signal tapering around the sea-floor reflected arrival helps to attenuate the bias due to the inaccuracies of the elastic model. The estimated reflection coefficient obtained for the real data didn't differ significantly from -1 and hence are not shown here. 

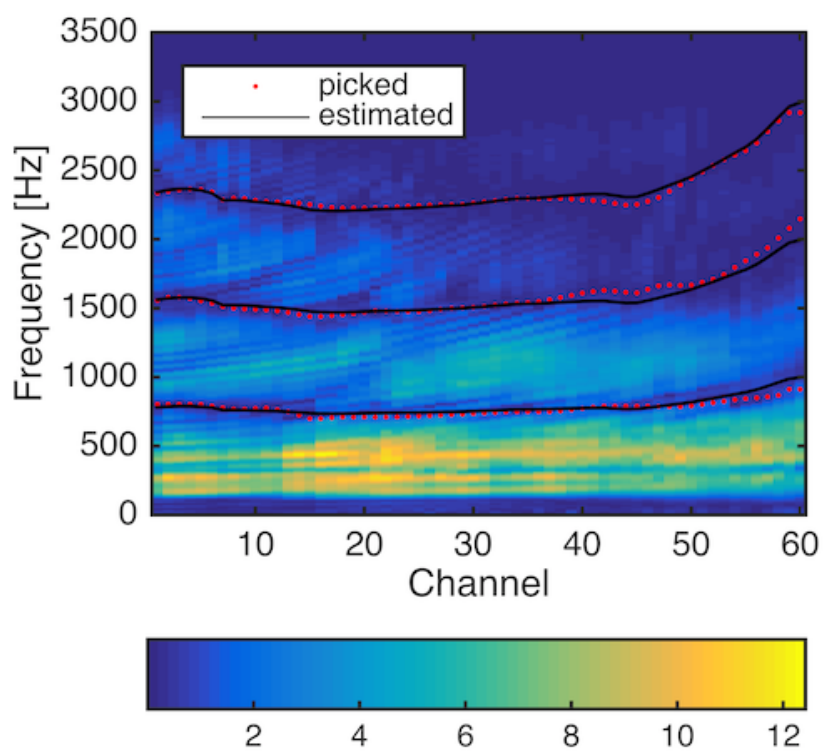

(a)

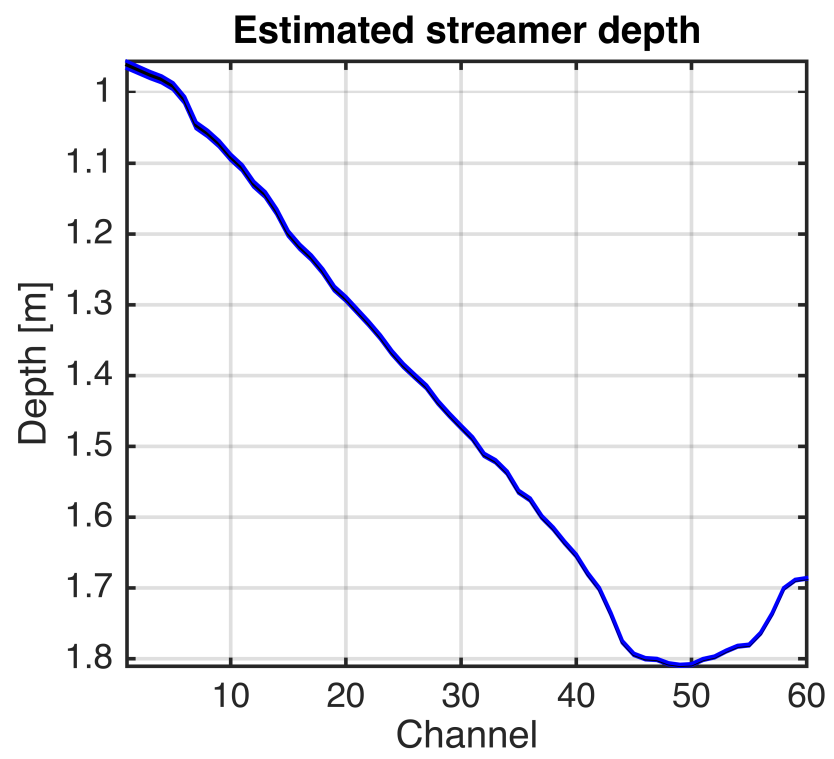

(b)

Figure A1. Streamer depth estimation. In panel a, the frequency-offset power spectrum with overlaid the picked and the predicted null frequencies; the latter are computed using Eq. A.7 from the inverted streamer depth shown in panel $b$. Note that the sagging receivers' depth profile and the uplift in the farther channels due to the use of a tail-buoy. 


\section{References}

Aki, K. \& Richards, P., 2002. Quantitative Seismology, University Science Books.

Asnaashari, A., Brossier, R., Garambois, S., Audebert, F., Thore, P., \& Virieux, J., 2012. Regularized seismic full waveform inversion with prior model information, Geophysics, 78(2), 25-36.

Aster, C., Borchers, B., \& Thurber, C., 2005. Parameter estimation and inverse problems, Elsevier Academic Press.

Aytun, K., 1999. The footsteps of the receiver ghost in the FK domain, Geophysics, 64(5), 16181626.

Bozdag, E., Trampert, J., \& Tromp, J., 2011. Misfit functions for full waveform inversion based on instantaneous phase and envelope measurements, Geophysical journal international, 185, $845-870$.

Bull, J., Quinn, R., \& Dix, J., 1998. Reflection coefficient calculation from marine high-resolution seismic reflection (chirp) data and application to an archelogical case study, Marine Geophysical Researches, 20, 1-11.

Bunks, C., Saleck, F. M., Saleski, S., \& Chavent, G., 1995. Multiscale seismic waveform inversion, Geophysics, 60, 1457-1473.

Burden, R. L. \& Faires, J. D., 1985. Numerical Analysis, 2.1 The Bisection Algorithm, PWS Publishers.

Cevatoglu, M., Bull, J., Vardy, M., Gernon, T., Wright, I., \& Long, D., 2015. Gas migration pathways, controlling mechanisms and changes in sediment acoustic properties observed in a controlled sub-seabed CO2 release experiment, International Journal of Greenhouse Gas Control.

Dagnino, D., Salláres, V., \& Ranero, C. R., 2014. Scale and parameter-adaptive model-based gradient pre-conditioner for elastic full-waveform inversion, Geophyscal journal international, 198, 1130-1142.

Debski, W. \& Tarantola, A., 1995. Information on elastic parameters obtained from the amplitudes of reflected waves, Geophysics, 60, 1426-1436.

Dey, A. \& Lines, L. R., 1998. Seismic source wavelet estimation and the random reflectivity 
assumption, Research report 10, 21-1-21-28., CREWES.

Fatti, J., Smith, G., Vail, P., Strauss, P., \& Levitt, P., 1994. Detection of gas sandstone reservoirs using AVO analysis: A 3d seismic case history using the geostack techcnique, Geophysics, 59(9), 1362-1376.

Fichtner, A., 2011. Full Seismic Waveform Modelling and Inversion, Springer.

Fichtner, A., Kennet, B. L. N., Igel, H., \& Bunge, H., 2008. Theoretical background for continental- and global-scale full-waveform inversion in the time-frequency domain, Geophyscal journal international, 175, 665-685.

Fuchs, K. \& Müller, G., 1971. Computation of synthetic seismograms with the reflectivity method and comparison with observations, Geophysical Journal Research Astronomical Society, 23, $417-433$.

Gholami, Y., Brossier, R., Operto, S., Prieux, V., Ribodetti, A., \& Virieux, J., 2013. Which parameterization is suitable for acoustic vertical transverse isotropic full waveform inversion? part 2: Synthetic and real data case studies from valhall, Geophysics, 78, 107-124.

Gulick, S., Christeson, G., Barton, P., Grieve, R., Morgan, J., \& Urrutia-Fucugauchi, J., 2013. Geophysical characterization of the chicxulub impact crater, Reviews of Geophysics, 51, 31-52. Hamilton, E., 1970. Sound velocity and related properties of marine sediments, North Pacific, Journal of Geophysical Research, 75, 4423-446.

Holland, C. \& Dettmer, J., 2013. In situ sediment dispersion estimates in the presence of discrete layers and gradient, The journal of the acoustical society of America, 133(1).

Igel, H., Djikpesse, H., \& Tarantola, A., 1996. Waveform inversion of marine reflection seismograms for p impedance and poisson's ration, Geophyscal journal international, 124, 363-371.

Jannane, M., 1989. Short note. wavelength of earth structures that can be resolved from seismic reflection data, Geophysics, 54(7), 906-910.

Jimenez-Tejero, C., Dagnino, D., Salláres, V., \& Ranero, C., 2015. Comparative study of objective functions to overcome noise and bandwidth limitations in full waveform inversion, Geophyscal journal international, 203, 632-645.

Jin, S., Madariaga, R., Virieux, J., \& Lambaré, G., 1992. Two-dimensional asymptotic iterative 
elastic inversion, Geophysical journal international, 108, 575-588.

Joo, Y., Seol, S., \& Byun, J., 2012. Acoustic full-waveform inversion of surface seismic data using the gauss-newton method with active constraint balancing, Geophysical prospecting, $\mathbf{6 1}$, $166-182$.

Kamei, R. \& Pratt, R., 2013. Inversion strategy for viscoacoustic waveform inversion, Geophysical journal international, 194, 859-884.

Koefoed, O., 1955. On the effect of poisson's ratio of rock strata on the reflection coefficient of plane waves, Geophysical prospecting, 3(4), 381-387.

Kormendi, F. \& Dietrich, M., 1991. Nonlinear waveform inversion of plane-wave seismograms in stratified elastic media, Geophysics, 56(5), 664-674.

Kwon, T., Seol, S., \& Byun, J., 2015. Efficient full-waveform inversion with normalized planewave data, Geophysical journal international, 201, 53-60.

Lailly, P., 1983. The seismic inverse problem as a sequence of before stack migrations, in Conference on Inverse Scattering: Theory and Application, pp. 206-220, Soc. Ind. Apl. Math., Philadelphia.

Lee, K. H. \& Kim, H. J., 2003. Source-independent full waveform inversion of seismic data, Geophysics, 68, 2010-2015.

Malinkowski, M., Operto, S., \& Ribodetti, A., 2011. High-resolution seismic attenuation imaging from wide-aperture onshore data by visco-acoustic frequency-domain full-waveform inversion, Geophyscal journal international, 186, 1179-1204.

Mallick, S. \& Adhikari, S., 2015. Amplitude variation with offset and pre-stack waveform inversion: a direct comparison using a real data example from the Rock Springs Uplift, Wyoming, USA, Geophysics, 80(2), B45-B59.

Mallick, S. \& Dutta, N., 2002. Shallow water flow prediction using prestack waveform inversion of conventional 3d seismic data and rock modeling, The Leading Edge, 21, 675-680.

Mavko, G., Mukerji, T., \& Dvorkin, J., 2009. The rock physics handbook, Cambridge U.P.

Menke, W., 1989. Geophysical data analysis and inverse problems, Academic Press INC.

Mora, P., 1980. Inversion = migration + tomography, Geophysics, 54(12), 1575-1586. 
Morgan, J., Warner, M., Bell, R., Ashley, J., Barnes, D., Little, R., Roele, K., \& Jones, C., 2013. Next-generation seismic experiments: wide-angle, multi-azimuth, three-dimensional, full-waveform inversion, Geophyscal journal international, 195(3), 1657-1678.

Nocedal, J. \& Wright, S. J., 2006. Numerical optimization, Springer.

Operto, S., Prieux, Y., Ribodetti, A., Brossier, R., Metivier, L., \& J.Virieux, 2013. A guided tour of multiparameter full waveform inversion with multicomponent data, The Leading Edge, pp. 936-947.

Ostrander, W., 1984. Plane-wave reflection coefficients for gas sands at nonnormal angles of incidence, Geophysics, 49(10).

Pinson, L., 2009. Derivation of Acoustic and Physical Properties from High-Resolution Seismic Reflection Data, Thesis for the degree of doctor of philosophy, School of Ocean and Earth Sciences, University of Southampton.

Pinson, L., Henstock, T., Dix, J., \& Bull, J., 2008. Estimating quality factor and mean grain size of sediment from high-resolution seismic data, Geophysics, 73, G19-G28.

Pratt, R., Plessix, E., \& Mulder, W., 1998. Gauss-newton and full newton methods in frequencyspace seismic waveform inversion, Geophysical journal international, 133, 341-362.

Richardson, M. \& Briggs, K., 1993. On the use of acoustic impedance values to determine sediment properties, in Acoustic classification and mapping of the seabed, vol. 15.

Riedel, M. \& Theilen, F., 2001. AVO investigations of shallow marine sediments, Geophysical prospecting, 49, 198-212.

Rodi, W. L., 1976. A technique for improving the accuracy of finite element solutions for magnetotelluric data, Geophysical Journal Research Astronomical Society, 44, 483-506.

Ruthenford, S. \& Williams, R., 1989. Amplitude-versus-offset variations in gas sands, Geophysics, 54(6), 680-688.

Schmidt, H. \& Jensen, F., 1985. A full wave solution for propagation in multilayered viscoelastic media with application to gaussian beam reflection at fluid-solid interfaces., Journal of the Acoustic Society America.

Schmidt, H. \& Tango, G., 1986. Efficient global matrix approach to the computation of synthetic 
seismograms., Geophysical Journal Research Astronomical Society.

Sheen, D., Tuncay, K., Baag, C., \& Ortoleva, P. J., 2006. Time domain gauss-newton seismic waveform inversion in elastic media, Geophyscal journal international, 167, 1373-1384.

Shin, C., Yoon, K., Marfurt, K. J., Park, K., Yang, D., Lim, H. Y., Chung, S., \& Shin, S., 2001. Efficient calculation of a partial-derivative wavefield using reciprocity for seismic imaging and inversion, Geophysics, 66, 1856-1863.

Silverton, A., Warner, M., Morgan, J., \& Umpleby, A., 2015. Offset-variable density improves acoustic full-waveform inversion: a shallow marine case study, Geophysical prospecting, pp. 1-14.

Stoker, M., Bradwell, T., Howe, J., Wilkinson, I., \& McIntyre, K., 2009. Lateglacial ice-cap dynamics in nw scotland: evidence from the fjords of the summer isles region., Quaternary Science Reviews, 28, 3161-3184.

Sun, D., Jiao, K., Cheng, J., \& Vigh, D., 2015. Compensating for source and receiver ghost effects in full waveform inversion and reverse time migration for marine streamer data, Geophyscal journal international, 201, 1507-1521.

Taner, M., Koehler, F., \& Sheriff, R., 1979. Complex seismic trace analysis, Geophysics, 44, $1041-1063$.

Tarantola, A., 1984. Inversion of seismic reflection data in the acoustic approximation, Geophysics, 49(8), 1259-1266.

Tarantola, A., 1986. A strategy for nonlinear inversion of seismic reflection data, Geophysics, 51(10), 1893-1903.

Ulrych, T., 1971. Application of homomorphic deconvolution to seismology, Geophysics, 36(4), $650-660$.

Vanneste, M., L'Heureux, J., Brendryen, J., Baeten, N., Larberg, J., Vardy, M., Steiner, A., Morgan, E., Forsberg, E., Kvalstad, C., Chand, T., et al., 2012. Assessing offshore geohazards: a multidisciplinary reasearch intiative to understand shallow landslides and their dynamics in coastal and deepwater environments, Norway., Advances in Natural and Technological Hazard Reseach, 31, 29-41. 
Vardy, M., 2015. Deriving shallow-water sediment properties using post-stack acoustic impedance inversion, Near Surface Geophysics, 13, 143-154.

Vardy, M., L'Heureux, J.-S., Vanneste, M., Longva, O., Steiner, A., Forsber, C., \& j. Brendryen, 2012. Multidisciplinary investigation of a shallow near-shore landslide, Finneidfjord, Norway, Near Surface Geophysics, 10, 267-277.

Verbeek, N. \& McGee, T. M., 1995. Characteristics of high-resolution marine reflection profiling sources, Journal of Applied Geophysics, 33, 251-269.

Verschur, D., Berkhout, A., \& Wapenaar, C., 1992. Adaptive surface-related multiple elimination, Geophysics, 57, 1166-1177.

Virieux, J. \& Operto, S., 2009. An overview of full-waveform inversion in exploration geophysics., Geophysics, 74(6).

Xu, Y., Gardner, G. H. F., \& McDonald, J. A., 1993. Some effects of velocity variation on AVO and its interpretation, Geophysics, 58(9), 1297-1300. 